\title{
Piezo1 regulates calcium oscillations and cytokine release from astrocytes
}

María Velasco-Estevez ${ }^{1}$, Sara O. Rolle ${ }^{2,3}$, Myrthe Mampay ${ }^{4}$, Kumlesh K. Dev ${ }^{1}$, Graham K. Sheridan ${ }^{4,5} \S$

${ }^{1}$ Drug Development, Department of Physiology, School of Medicine, Trinity College Dublin, Dublin, Ireland.

${ }^{2}$ Department of Bioengineering, Imperial College London, London, UK.

${ }^{3}$ Francis Crick Institute, London, UK.

${ }^{4}$ School of Pharmacy and Biomolecular Sciences, University of Brighton, Brighton, UK.

${ }^{5}$ School of Life Sciences, Queen's Medical Centre, University of Nottingham, Nottingham UK.

\section{${ }^{\S}$ Correspondence should be addressed to:}

Dr. Graham Sheridan

School of Life Sciences, Queen's Medical Centre, University of Nottingham, Nottingham UK.

Tel: +44 1237642030

Email: Graham.Sheridan@nottingham.ac.uk

Running title: Astrocytic Piezo1 and neuroinflammation.

Key Words: Astrocytes, Calcium oscillations; Lipopolysaccharide; Mechanosensitive ion channel, Piezo1; Pro-inflammatory cytokines.

\section{Financial Support}

This work was supported by an Irish Research Council PhD scholarship to M.V.E, a Crick PhD studentship to S.O.R, a University of Brighton PhD studentship to M.M, and a Pump Priming research grant to G.K.S from the University of Brighton. G.K.S is also grateful for funding from the Leverhulme Trust [RPG-2018-443]. 


\title{
Author contributions
}

G.K.S and K.K.D conceived the project and designed the research; M.V.E, M.M, and G.K.S performed the experiments; M.V.E, S.O.R, and G.K.S analysed the data; all authors discussed the results, contributed to the writing of the manuscript and approved the final version.

\section{Acknowledgements}

The authors wish to thank Sinead O'Sullivan for valuable technical assistance in the early stages of the project.

\section{Conflict of Interest Statement}

The authors declare no conflicts of interest and no competing financial interests.

\section{Data availability}

The data that support the findings of this study are available from the corresponding author upon reasonable request.

\begin{abstract}
Abbreviations
A $\beta 42$, Amyloid- $\beta$ 1-42 peptide; BSA, Bovine Serum Albumin; CNS, Central Nervous System; DMSO, Dimethyl sulfoxide; DMEM, Dulbecco's modified Eagle's medium; ER, Endoplasmic reticulum; FBS, Foetal Bovine Serum, GFAP, Glial Fibrillary Acidic Protein; HBSS, Hank's balanced salt solution; HRP, Horse radish peroxidase; IL, Interleukin; LPS, Lipopolysaccharide; PBS, Phosphate-buffered saline; PVDF, Polyvinylidene difluoride membrane; RIPA, radioimmunoprecipitation assay; SDS-PAGE, Sodium Dodecyl Sulfate Polyacrylamide Gel Electrophoresis; TNF $\alpha$, Tumour Necrosis Factor-alpha; RRID, Research Resource Identifier.
\end{abstract}

\section{Main Points}

- Activation of Piezo1 channels in LPS-stimulated astrocytes induces calcium influx and augments intracellular calcium oscillations

- Piezo1 activation inhibits the release of pro-inflammatory cytokines, IL-1 $\beta$ and $\mathrm{TNF} \alpha$, from LPS-stimulated astrocytes 


\begin{abstract}
Astrocytes are important for information processing in the brain and they achieve this by fine-tuning neuronal communication via continuous uptake and release of biochemical modulators of neurotransmission and synaptic plasticity. Often overlooked are their important functions in mechanosensation. Indeed, astrocytes can detect pathophysiological changes in the mechanical properties of injured, ageing or degenerating brain tissue. We have recently shown that astrocytes surrounding mechanically-stiff amyloid plaques upregulate the mechanosensitive ion channel, Piezo1. Moreover, ageing transgenic rats harbouring a chronic peripheral bacterial infection displayed enhanced Piezol expression in amyloid plaque-reactive astrocytes of the hippocampus and cerebral cortex. Here, we have shown that the bacterial endotoxin, lipopolysaccharide (LPS), also upregulates Piezo1 in primary mouse cortical astrocyte cultures in vitro. Activation of Piezo1, via the small molecule agonist Yodal, enhanced $\mathrm{Ca}^{2+}$ influx in both control and LPS-stimulated astrocytes. Moreover, Yoda1 augmented intracellular $\mathrm{Ca}^{2+}$ oscillations but decreased subsequent $\mathrm{Ca}^{2+}$ influx in response to adenosine triphosphate (ATP) stimulation. Neither blocking nor activating Piezol affected cell viability. However, LPS-stimulated astrocyte cultures exposed to the Piezol activator, Yoda1, migrated significantly slower than reactive astrocytes treated with the mechanosensitive channel-blocking peptide, GsMTx4. Furthermore, our data show that activating Piezol channels inhibits the release of cytokines and chemokines, such as IL-1 $\beta, \mathrm{TNF} \alpha$ and fractalkine ( $\left.\mathrm{CX}_{3} \mathrm{CL} 1\right)$, from LPS-stimulated astrocyte cultures. Taken together, our results suggest that astrocytic Piezol upregulation may act to dampen neuroinflammation and could be a useful drug target for neuroinflammatory disorders of the brain.
\end{abstract}




\section{Introduction}

Astrocytes are the most abundant glial cell type in the brain and form interconnected neuron-glial networks that are exquisitely sensitive sensors and modulators of neuronal activity (Copeland et al., 2017; Fellin et al., 2004; Serrano, Haddjeri, Lacaille, \& Robitaille, 2006). Astrocytes are also highly mechanosensitive cells (Blumenthal, Hermanson, Heimrich, \& Shastri, 2014; Moshayedi et al., 2010) and can detect changes in the mechanical properties of their surrounding microenvironment during disease of the central nervous system (CNS) or following traumatic injury (Maneshi, Sachs, \& Hua, 2015; Moshayedi et al., 2014). We have recently shown that astrocytes which wrap and engulf stiff amyloid plaques in the ageing rat brain upregulate expression of the mechanosensitive ion channel, Piezo1 (Velasco-Estevez et al., 2018). Piezo1 is a non-selective cation channel expressed on the outer cell membrane and is permeable to extracellular calcium $\left(\mathrm{Ca}^{2+}\right)$ influx (Gottlieb \& Sachs, 2012; Li et al., 2015). The calcium-mediated mechanotransduction signalling cascades activated by Piezol channel opening are important in a range of developmental and physiological processes including neuronal differentiation (Pathak et al., 2014), axon guidance (Koser et al., 2016), confinement-sensing and cell migration (Hung et al., 2016), neuronal regeneration (Song et al., 2019), shear stress-mediated vasodilation (Wang et al., 2016), and immune cell activation (Liu et al., 2018). However, the intracellular molecular mechanisms that underlie Piezol's role in many of the above cellular processes have yet to be fully elucidated. McHugh et al. (2010) have also shown that Piezol channels localise to the endoplasmic reticulum (ER) in epithelial cells where they can regulate integrin-mediated cell adhesion via calpain and talin activation. Therefore, Piezol channels can regulate intracellular $\mathrm{Ca}^{2+}$ concentrations via $\mathrm{Ca}^{2+}$ release from intracellular stores or through the formation of mechanically-gated ion channels in the outer cell membrane (Nourse \& Pathak, 2017). Interestingly, Piezo1 is also voltage sensitive (Moroni, Servin-Vences, Fleischer, Sanchez-Carranza, \& Lewin, 2018) and, therefore, its repertoire of known functions in CNS neurons and glial cells, both in health and disease, is likely to increase in the near future.

In endothelial cells, shear stress activates Piezo1-mediated $\mathrm{Ca}^{2+}$ influx leading to 
the release of adenosine triphosphate (ATP) (Wang et al., 2016). In turn, ATP can activate purinergic $G$ protein-coupled receptors, such as $\mathrm{P}_{2} \mathrm{Y}_{1}$ and $\mathrm{P} 2 \mathrm{Y}_{2}$, on neighbouring cells causing the upregulation of nitric oxide synthase (NOS) (Jacob, Perez Novo, Bachert, \& Van Crombruggen, 2013). Nitric oxide (NO) release from astrocytes acts as an important neuromodulator in the brain and can induce further gliotransmitter release, including glutamate (Bal-Price, Moneer, \& Brown, 2002). ATP and glutamate have a wide range of functions at the synapse and can initiate $\mathrm{Ca}^{2+}$ oscillations and $\mathrm{Ca}^{2+}$ waves in astrocytes (Leybaert \& Sanderson, 2012; Scemes \& Giaume, 2006), thus modulating long-range communication and information processing through astrocyte-neuron networks. ATP can also regulate the release of neuromodulators from astrocytes, such as cytokines that fine-tune local neurotransmission and synaptic plasticity processes (Adzic et al., 2017; Nishizaki, 2004). Interestingly, astrocytic Piezo1 expression was relatively high in the cortex and hippocampus of transgenic Alzheimer's rats (TgF344-AD) that harboured a chronic urinary tract infection (UTI) of Escherichia coli (E. coli). This suggests that raised levels of cytokines, chemokines and neuroinflammation, which often develops in the elderly as a result of chronic peripheral immune activation (Chesnokova, Pechnick, \& Wawrowsky, 2016; Simen, Bordner, Martin, Moy, \& Barry, 2011), may lead to the enhanced expression of Piezo1 in amyloid plaquereactive astrocytes (Velasco-Estevez et al., 2018). However, whether this upregulation of mechanosensitive $\mathrm{Ca}^{2+}$ permeable channels in the ageing Alzheimer's disease brain is neuroprotective, or instead enhances neuro-damaging signalling cascades, is as yet unknown.

Here, we used an in vitro murine-derived primary astrocyte culture model to examine the effects of activating Piezo1 on several key functional parameters including, 1) cytosolic $\mathrm{Ca}^{2+}$ oscillations, 2) ATP-induced $\mathrm{Ca}^{2+}$ influx, 3) astrocyte migration, and 4) cytokine and chemokine release from control and LPS-stimulated astrocyte cultures. The aim was to investigate if upregulation of Piezo1 in reactive astrocytes exacerbates neuroinflammation or whether Piezol expression in LPSstimulated astrocytes could be an innate neuroprotective response to disease pathogenesis in the inflamed brain. 


\section{Materials and Methods}

\section{Ethics statement}

All experiments involving animals, and Schedule 1 protocols used to obtain brain tissue, were approved by the Animal Welfare and Ethical Review Bodies (AWERB committees) of the University of Brighton and Trinity College Dublin. This study was conducted in accordance with the principles of the Basel Declaration and adhered to the legislation detailed in the UK Animals (Scientific Procedures) Act 1986 Amendment Regulations (SI 2012/3039). All efforts were taken to maximise animal welfare conditions and to reduce the number of animals used in accordance with the European Communities Council Directive of September $20^{\text {th }}, 2010$ (2010/63/EU).

\section{Mouse cortical astrocyte cultures}

Mixed glial cell cultures were prepared from cerebral cortical tissue from postnatal day one (P1) male and female wild-type C57BL/6 mice (RRID: IMSR_JAX:000664) bred in the BioResources Units of Trinity College Dublin and the University of Brighton. Two mouse cortices were used per T75 flask preparation. Mixed glial cells were cultured at $37^{\circ} \mathrm{C}$ and $5 \% \mathrm{CO}_{2}$ in a humidified incubator for 14 days in Dulbecco's modified Eagle's medium/F12 (DMEM/F12; Hyclone SH30023) supplemented with $10 \%$ foetal bovine serum (FBS; Labtech, FB-1090) and 1\% penicillin/streptomycin (Sigma, P4333), as previously described (Healy et al., 2013; O'Sullivan, Velasco-Estevez, \& Dev, 2017). After 14 days in vitro (DIV), T75 flasks containing confluent glial cell cultures were shaken for $2 \mathrm{~h}$ at $200 \mathrm{rpm}$ using an orbital shaker (VWR, Microplate shaker Model No. 980131UK) to eliminate most of the microglia and oligodendrocytes from the glial monolayer. The remaining enriched astrocyte culture was exposed to $0.5 \%$ trypsin for $5 \mathrm{~min}$ to cause detachment of the glial monolayer and cells were then re-plated in a new T75 flask to further purify the astrocyte culture and eliminate loosely attached microglia. Once the re-plated cells were $80 \%$ confluent, they were again exposed to $0.5 \%$ trypsin, detached and re-plated in different cell culture substrates 
for the various experiments described below. Using this method, enriched astrocyte cultures are $>95 \%$ pure, as previously described (Healy et al., 2013).

\section{Immunocytochemistry}

After each pharmacological treatment, cells were fixed in $10 \%$ formalin solution (Sigma, F1635) for $5 \mathrm{~min}$ on ice. Cells were blocked for $18 \mathrm{~h}$ at $4^{\circ} \mathrm{C}$ in blocking solution containing 1\% bovine serum albumin (BSA; Santa-Cruz, sc-2323) and 0.1\% Triton X-100 (Sigma, T8787). Primary antibodies were diluted in blocking solution and incubated for $18 \mathrm{~h}$ at $4^{\circ} \mathrm{C}$. Cells were washed with PBS containing $0.1 \%$ Triton $\mathrm{X}-100$ followed by incubation with secondary antibodies diluted in blocking solution at $22^{\circ} \mathrm{C}$ for one hour in the dark. After several washes, coverslips were mounted on a microscope slide (Clarity, C361) with antifade reagent (ThermoFisher, S36936). Primary antibodies used were: Piezo1 (Abcam, ab128245; RRID: AB_11143245; rabbit polyclonal, dilution 1/500) and GFAP (Abcam, ab4674; RRID: AB_304558; chicken polyclonal, dilution 1/1,000). Secondary antibodies used were biotinylated anti-rabbit (ThermoFisher, A24535; RRID: AB_2536003; dilution 1/1,000), avidin Alexa ${ }^{\circledR} 488$ conjugate (ThermoFisher, A21370; dilution 1/1,000) and Alexa ${ }^{\circledR} 633$ anti-chicken (Alexa, A21103; RRID: AB_2535756; dilution 1/1,000). Imaging was performed using a Leica TCS SP8 confocal microscope.

\section{Quantitative Reverse-transcription PCR (RT-qPCR)}

RNA extraction was performed using the RNeasy mini kit (QIAGEN, \#74104), following the manufacturer's instructions. The quantity and quality of mRNA was measured by spectrophotometric analysis using a nanodrop (ThermoFisher, NanoDrop ${ }^{\mathrm{TM}}$ One/One ${ }^{\mathrm{c}}$ microvolume). RNA concentration was quantified using the optical density (OD) at $260 \mathrm{~nm}$. The $260 / 280 \mathrm{~nm}$ ratios were used as a measure of quality and only samples with values between $1.8-2.0$ were considered acceptable. Reverse transcription of the mRNA was performed using the QuantiNova Reverse Transcription Kit (QIAGEN, \#205411) in a ${ }^{3}$ Prime thermocycler (TECHNE, 3PRIMEG/02, serial no. *32288*). The PCR master mix 
was obtained by mixing SYBR green dye (QIAGEN, \#1054596), Quantitect ${ }^{\circledR}$ Primer of mouse Piezo1 (Mm_Piezo1_3_SG; QT01199142) or $\beta$-actin (Mm_Actb_1_SG; QT00095242) and the cDNA template. The reaction was performed in a Rotor-Gene Q 5plex (QIAGEN, \#90158) with PCR cycles of denaturation at $95^{\circ} \mathrm{C}$ for 5 seconds and annealing/extension at $60^{\circ} \mathrm{C}$ for 10 seconds, 40 times.

\section{Western-Blot}

Mouse astrocyte protein samples were obtained by scraping the cell monolayer in RIPA buffer. Samples were run in a $6 \%$ SDS-PAGE electrophoresis gel and transferred to PVDF membranes (Millipore, IPVH00010) by wet transfer (buffer composition: $2.5 \mathrm{mM}$ Tris, $19.2 \mathrm{mM}$ Glycine and 5\% methanol, $\mathrm{pH}$ 8.3) at constant $70 \mathrm{~V}$ for $3 \mathrm{~h}$ on ice. Membranes were then blocked in PBS containing 5\% BSA and $0.05 \%$ Tween-20 for $2 \mathrm{~h}$ at $22^{\circ} \mathrm{C}$ and incubated with primary antibodies that recognise Piezo1 (Abcam, ab128245; RRID: AB_11143245; dilution 1/300 in blocking buffer) or $\alpha$-actin (Abcam, ab3280; RRID: AB_303668; dilution 1/2,000 in blocking buffer) for $18 \mathrm{~h}$ at $4^{\circ} \mathrm{C}$. After several PBS and PBS-Tween $0.05 \%$ washes, membranes were incubated with a goat anti-rabbit HRP-conjugated secondary antibody (GE Healthcare, NA934; RRID: AB_772206; dilution 1/5,000 in blocking buffer) or goat anti-mouse secondary antibody (Sigma, A8924; RRID: AB_258426; dilution $1 / 5,000$ in blocking buffer) for $2 \mathrm{~h}$ at $22^{\circ} \mathrm{C}$. Several washes were then performed and PVDF membranes were developed using a chemiluminescent HRP substrate (Millipore, WBKLS0500).

\section{MTT assay}

Astrocytes were seeded in 96-well plates (Corning) at a density of 15,000 cells/well and grown for $48 \mathrm{~h}$ in DMEM/F12 medium containing 10\% FBS. They were then serum-starved for $3 \mathrm{~h}$ prior to stimulation with LPS $(100 \mathrm{ng} / \mathrm{ml})$ alone or in combination with GsMTx4 $(500 \mathrm{nM})$ or Yoda1 $(10 \mu \mathrm{M})$ for $48 \mathrm{~h}$. Following each treatment, the cell culture medium was removed and stored for ELISA assays and 
replaced with $100 \mu \mathrm{L}$ of fresh medium supplemented with $1 \mathrm{mg} / \mathrm{mL} 3-(4,5-$ dimethylthiazol-2-yl)-2,5-diphenyltetrazolium bromide (MTT) (Invitrogen, M6494) for $3 \mathrm{~h}$ at $37^{\circ} \mathrm{C}$. Next, $75 \mu \mathrm{L}$ of culture medium was removed and $50 \mu \mathrm{L}$ of dimethyl sulfoxide (DMSO) was added. Astrocytes were incubated for $10 \mathrm{~min}$ at $37^{\circ} \mathrm{C}$ and the absorbance in each well was read at a wavelength of $540 \mathrm{~nm}$.

\section{JC-1 assay}

Changes in mitochondrial potential were measured using a commercially available JC-1 assay kit (Abcam, ab113850) following the manufacturer's instructions. Briefly, astrocytes were seeded in a 96-well plate at a density of 15,000 cells/well and treated as described above for the MTT assay. 30 min prior to the end of each treatment, $10 \mu \mathrm{M} \mathrm{JC}-1$ dye was added to each well at a final concentration of $5 \mu \mathrm{M}$. After $30 \mathrm{~min}$, the wells were washed twice with dilution buffer. The excitation wavelength was set at $535 \pm 17.5 \mathrm{~nm}$ and the emission at $590 \pm 17.5 \mathrm{~nm}$. Aggregate emission was measured in a BioTek synergy HT microplate reader (BioTek, VT, USA).

\section{ELISA assay}

Cytokine and chemokine release from astrocytes were quantified using commercially available duo-set ELISA kits (R\&D Systems) for mouse IL-6 (DY406), TNF- $\alpha$ (DY410), IL-1 $\beta /$ IL-1F2 (DY401) and CX 3 CL1 (DY472). Briefly, 96 well MicroWell ${ }^{\mathrm{TM}}$ MaxiSorp ${ }^{\mathrm{TM}}$ flat bottom plates (Sigma, 442404) were coated with the capture antibody diluted in PBS for $18 \mathrm{~h}$ at $4^{\circ} \mathrm{C}$. Plates were then washed in $0.5 \%$ Tween-20 in PBS and blocked with the appropriate reagent diluent for $1 \mathrm{~h}$ at $22^{\circ} \mathrm{C}$. The blocking buffer was then removed and the astrocyte culture medium and recombinant protein standards were diluted, added to the wells and incubated for $2 \mathrm{~h}$ at $22^{\circ} \mathrm{C}$. The wells were then washed and the detection antibody was added for $2 \mathrm{~h}$ at $22^{\circ} \mathrm{C}$. The detection antibody was then washed off and a solution containing streptavidin-HRP secondary antibody was added for 20 min and kept in the dark. The secondary antibody solution was washed off and the substrate solution 
was added (DY999, R\&D Systems) and incubated for $20 \mathrm{~min}$. The reaction was stopped by adding $2 \mathrm{~N} \mathrm{H}_{2} \mathrm{SO}_{4}$ to the plate and absorbance was immediately read at $450 \mathrm{~nm}$ with wavelength correction at $570 \mathrm{~nm}$.

\section{Cell migration assay}

A $500 \mu \mathrm{m}$ wide strip of silicone (silicone sheet of 0.0005 " NRV G/G 12"x12", SM11074036) was placed down the middle of each well of a 12 well-plate. Astrocytes were then seeded onto each well and grown in supplemented DMEM/F12 media until 90\% confluent. Cells were then serum-starved and treated with $100 \mathrm{ng} / \mathrm{mL}$ LPS (Sigma, L4391) with or without $10 \mu \mathrm{M}$ Yoda1 (Tocris, \#5586) or $500 \mathrm{nM}$ GsMTx4 (Alomone, STG-100) for $24 \mathrm{~h}$. The silicone strip was then carefully removed and the $500 \mu \mathrm{m}$ “wound area” was imaged at 'time zero' using a Nikon Eclipse Ti-U microscope with 10x magnification objective and a Nikon digital sight camera connected to the NIS-Elements BR 3.2 software package (Nikon, Japan). The wound area was imaged again $48 \mathrm{~h}$ post-silicone removal and the change in the width of the area containing no cells was calculated using ImageJ software (http://imagej.nih.gov/ij).

\section{Live-cell calcium imaging}

Astrocytes were seeded onto $25 \mathrm{~mm}$ tissue culture plastic coverslips (Sarstedt, \#83.1840) in a 6 well-plate and grown for $72 \mathrm{~h}$ in supplemented DMEM/F12 until $70 \%$ confluent. Cells were then serum-starved for $3 \mathrm{~h}$ prior to treatment with or without $100 \mathrm{ng} / \mathrm{mL}$ LPS for $24 \mathrm{~h}$. After treatment, cells were incubated with $3 \mu \mathrm{M}$ Cal-520AM dye (Abcam, ab171868) in Hank's balanced salt solution (HBSS; no phenol red) supplemented with $10 \mathrm{mM}$ glucose and $25 \mathrm{mM}$ HEPES for $90 \mathrm{~min}$ at $37^{\circ} \mathrm{C}$, followed by $30 \mathrm{~min}$ at $22^{\circ} \mathrm{C}$. Cells were protected from bright light at all times. Coverslips were then placed into the Attofluor ${ }^{\mathrm{TM}}$ acquisition chamber (Invitrogen) with supplemented HBSS or HBSS minus $\mathrm{Ca}^{2+}$ and $\mathrm{Mg}^{2+}\left(\mathrm{HBSS}^{-/-}\right)$. Time-lapse $\mathrm{Ca}^{2+}$ imaging was performed at a rate of 0.77 frames per second. Following a 30 second baseline, cells were stimulated with either HBSS or $10 \mu \mathrm{M}$ Yoda1 for 240 seconds and stimulated further with $50 \mu \mathrm{M}$ ATP for 180 seconds. 
Extracellular $\mathrm{Ca}^{2+}$ imaging experiments were performed with standard HBSS and intracellular $\mathrm{Ca}^{2+}$ imaging experiments were performed with $\mathrm{HBSS}$ minus $\mathrm{Ca}^{2+}$ and $\mathrm{Mg}^{2+}\left(\mathrm{HBSS}^{--}\right)$. Images were acquired using a Leica SP5 confocal microscope and a $20 \times$ magnification objective.

\section{Calcium imaging analysis}

Time-lapse $\mathrm{Ca}^{2+}$ imaging experiments were saved as Leica .lif files and exported as individual RAW 8 bit tif images (349 in total per experiment). The maximum intensity projection image was used to create a binary mask allowing for segmentation of the monolayer of astrocytes into individual cells. Image analysis was performed using the R programming environment and EBImage package (Pau, Fuchs, Sklyar, Boutros, \& Huber, 2010). Two image segmentation steps were performed. The first identified individual astrocyte nuclei and the second was performed to segment the cytosol of each astrocyte from one another. Nuclear and cytosolic $\mathrm{Ca}^{2+}$ responses for each astrocyte were analysed separately, but both provided very similar results and so only the cytosolic $\mathrm{Ca}^{2+}$ imaging quantification is presented here. Absolute changes in cytosolic $\mathrm{Ca}^{2+}$ fluorescence intensities were measured for each cell over a $7 \mathrm{~min} 30$ second time period and were then normalised to the baseline fluorescence intensity values for each cell $\left[\Delta \mathrm{F} / \mathrm{F}_{0}\right]$, where $\mathrm{F}_{0}$ is the average fluorescence intensity of frames 5-15. This converted the absolute changes in cytosolic $\mathrm{Ca}^{2+}$ fluorescence intensity to relative changes in $\mathrm{Ca}^{2+}$ fluorescence per cell. There were four treatment groups per experiment, 1) Control/HBSS, 2) Control/Yoda, 3) LPS/HBSS, and 4) LPS/Yoda. Experiments were conducted either in the presence (Extracellular $\mathrm{Ca}^{2+}$ ) or absence (Intracellular $\mathrm{Ca}^{2+}$ ) of extracellular $\mathrm{Ca}^{2+}$ and $\mathrm{Mg}^{2+}$ using either Control or LPS pre-treated astrocytes. Extracellular $\mathrm{Ca}^{2+}$ experiments were repeated $\mathrm{n}=8$ with 3 technical replicates per treatment group, i.e. the $\mathrm{Ca}^{2+}$ imaging experiments were repeated on 8 separate days using astrocyte cultures from 8 different mouse litters and 3 x $25 \mathrm{~mm}$ coverslips of cells were imaged per group during each live-cell imaging session. Intracellular $\mathrm{Ca}^{2+}$ experiments were repeated $\mathrm{n}=6$ with 3 technical replicates per group. In total, the number of astrocytes analysed per treatment group were as follows: For 
Extracellular $\mathrm{Ca}^{2+}$ experiments, 1) Control/HBSS $\left.=4,286,2\right)$ Control/Yoda $=$ $4,023,3) \mathrm{LPS} / \mathrm{HBSS}=3,980$, and 4) LPS/Yoda $=3,915$. For Intracellular $\mathrm{Ca}^{2+}$ experiments, Control/HBSS $\left.{ }^{-/-}=3,866,2\right)$ Control/Yoda $\left.{ }^{-/-}=3,967,3\right) \mathrm{LPS} / \mathrm{HBSS}^{-/-}$ $=3,069$, and 4) $\mathrm{LPS} /$ Yoda $^{-/-}=3,290$. In Figures 3 and 5, the relative changes in cytosolic $\mathrm{Ca}^{2+}$ fluorescence are expressed as the median value \pm interquartile range (IQR). Moreover, the first 2 min (early) and the last 2 min (late) of HBSS or Yoda1 perfusion are also displayed in Figures $3 \mathrm{~A}$ and $5 \mathrm{~A}$ as the mean change in cytosolic $\mathrm{Ca}^{2+}$ fluorescence \pm the standard error of the mean.

\section{Statistical analysis}

All statistical analysis was performed using GraphPad Prism 7 (GraphPad Prism ${ }^{\circledR}$, RRID:SCR_002798). Assessment of the normality of data was carried out using the column statistics function and D'Agostino criteria before any further statistical test was performed. When only two groups were compared, a paired Student t-test was performed. In cases when three or more groups needed to be compared, repeated measures one-way or two-way ANOVAs were performed and post-hoc tests were applied with $\mathrm{p}$ value adjustments using Bonferroni or Holm-Sidak methods. The analysis of proportions was carried out using chi-squared $\left(\chi^{2}\right)$ tests in conjunction with the Bonferroni correction for multiple comparisons. $\mathrm{P}$ values $<0.05$ were considered statistically significant. Further details of the statistical analyses performed are given in each figure legend and the results section. 


\section{Results}

\section{LPS induces Piezo1 expression in cortical astrocytes}

We have recently shown in a rat model of Alzheimer's disease (TgF344-AD) that hippocampal and cortical astrocytes surrounding amyloid plaques upregulate Piezo1 expression. Interestingly, astrocytic Piezo1 expression is enhanced in rats harbouring a chronic urinary tract infection with E. coli (Velasco-Estevez et al., 2018). Here, we show that mouse cortical astrocytes exposed to lipopolysaccharide (LPS) upregulate Piezo1 channel expression. The Gram-negative bacterial endotoxin LPS induces a strong immune reaction in animals and is an agonist of toll-like receptor 4 (TLR4) which is expressed by mouse astrocytes (Nakano et al., 2015). Treatment with $100 \mathrm{ng} / \mathrm{mL}$ LPS induced an inflammatory phenotype in primary cortical astrocytes (Fig. 1A-D) and caused an upregulation of Piezo1 channels after $24 \mathrm{~h}$ (Fig. 1E). Approximately $2-3 \%$ of astrocytes expressed low levels of Piezo1 under non-inflammatory conditions and this rose to $20-25 \%$ of astrocytes after $24 \mathrm{~h}$ LPS exposure (Fig. 1F). Interestingly, Piezo1 expression (measured as immunofluorescence intensity) decreased again after $48 \mathrm{~h}$ exposure to LPS (Fig. 1E), but the proportion of cells expressing low levels of Piezo1 remained above control (Fig. 1F). Piezol upregulation in astrocytes following $24 \mathrm{~h}$ exposure to LPS was confirmed by Western blot (Fig. 1G) and by PCR analysis of mRNA transcript levels (Fig. 1H).

\section{Piezo1 activation enhances calcium influx in LPS-stimulated astrocytes}

Piezo1 is a $\mathrm{Ca}^{2+}$ permeable cation channel and since LPS caused an increase in Piezo1 expression in astrocytes (Fig. 1), we next investigated the effects of Piezo1 activation on $\mathrm{Ca}^{2+}$ influx and $\mathrm{Ca}^{2+}$ oscillations in cortical astrocytes. Astrocyte cultures were pre-treated either with or without LPS $(100 \mathrm{ng} / \mathrm{mL})$ for $24 \mathrm{~h}$ and then loaded with Cal-520AM calcium dye (Fig. 2A). Time-lapse $\mathrm{Ca}^{2+}$ imaging was performed at a rate of 0.77 frames per second. After a $30 \mathrm{~s}$ baseline recording (23 frames), the Piezol agonist, Yoda1 $(10 \mu \mathrm{M})$, was perfused onto non-treated astrocyte cultures (i.e. Control/Yoda) causing an increase in cytosolic $\mathrm{Ca}^{2+}$ concentration in $48 \%$ of astrocytes (i.e. $52 \%$ of cells did not respond to Yoda1). 
Because Piezol channels can be activated by shear stress, HBSS was used to control for extracellular solution perfusion (i.e. Control/HBSS). $46 \%$ of cells responded to the addition of HBSS alone (i.e. showed one or more $\mathrm{Ca}^{2+}$ peaks) but the increases in cytosolic $\mathrm{Ca}^{2+}$ were significantly lower than for Yoda1 (Fig. 2B). Similarly, 45\% of cells in the LPS-stimulated astrocyte cultures responded to HBSS perfusion, whereas $60 \%$ of cells responded to Yodal ( $\chi^{2}$ test, $\left.\mathrm{p}<0.001\right)$ which caused much larger $\mathrm{Ca}^{2+}$ influxes (Fig. 2C). This was presumably due to the upregulation of Piezo1 channels in LPS-stimulated astrocytes (Fig. 1).

The median area under the curve (AUC with arbitrary fluorescence units; a.f.u.) was negative in response to HBSS, i.e. -5.2 a.f.u (-36.9, +41.4 IQR) suggesting a small net efflux of intracellular $\mathrm{Ca}^{2+}$ (Fig. 3A). Yoda1, on the other hand, induced an increase in $\mathrm{Ca}^{2+}$ influx, with the median AUC measured as +48.3 a.f.u (-3.2, +158.1 IQR). Interestingly, for control astrocytes perfused with HBSS or Yoda1 and for LPS-stimulated cultures perfused with HBSS, $9-10 \%$ of cells responded with two or more $\mathrm{Ca}^{2+}$ peaks (Fig. 3B) over the 4 min time-frame. In contrast, astrocytes pre-treated with LPS and perfused with Yoda1 displayed a doubling (20\%) in the proportion of cells that responded with two or more $\mathrm{Ca}^{2+}$ peaks $\left(\chi^{2}\right.$ test, $\left.\mathrm{p}<0.001\right)$, suggesting that Piezol increases $\mathrm{Ca}^{2+}$ oscillations in LPS-reactive astrocytes (Fig. $3 B)$.

Activation of Piezo1 in LPS-stimulated astrocytes modifies their response to ATP

After recording control and LPS-stimulated astrocyte $\mathrm{Ca}^{2+}$ responses to both HBSS and Yoda1 for $4 \mathrm{~min}$, all four groups of cells were then perfused with $50 \mu \mathrm{M}$ ATP. Time-lapse images were captured for a further $3 \min$ (140 frames) to determine the proportions of astrocytes that display ATP-induced $\mathrm{Ca}^{2+}$ oscillations. Control and LPS-stimulated astrocytes pre-perfused with HBSS displayed ATP-induced $\mathrm{Ca}^{2+}$ peaks that were larger in amplitude to those elicited by astrocytes pre-perfused with Yoda1 (Fig. 3C). This is because the Control/HBSS and LPS/HBSS groups displayed negative median AUC $\mathrm{Ca}^{2+}$ responses to HBSS perfusion and, therefore, had low basal $\mathrm{Ca}^{2+}$ levels upon the addition of ATP. This enabled them to respond 
with large ATP-induced $\mathrm{Ca}^{2+}$ peaks. The LPS/HBSS group showed the largest AUC $\mathrm{Ca}^{2+}$ response to ATP perfusion, i.e. +151.9 a.f.u (+84.2, +225.5 IQR). Interestingly, astrocytes that displayed the highest number of $\mathrm{Ca}^{2+}$ oscillations to Yoda1 (i.e. the LPS/Yoda1 group) responded with the lowest number of $\mathrm{Ca}^{2+}$ peaks following ATP perfusion, i.e. $26 \%$ of astrocytes in the LPS/Yoda1 group displayed 2 or more $\mathrm{Ca}^{2+}$ peaks whereas $45 \%$ of the Control/HBSS group showed ATP-induced $\mathrm{Ca}^{2+}$ oscillations $\left(\chi^{2}\right.$ test, $\left.\mathrm{p}<0.001\right)$ (Fig. 3D). Taken together, our data indicate that Yoda1 enhances $\mathrm{Ca}^{2+}$ oscillations in LPS-stimulated astrocytes (due to upregulation of Piezo1), but this increases basal $\mathrm{Ca}^{2+}$ levels and consequently reduces the proportion of cells that display $\mathrm{Ca}^{2+}$ oscillations in response to ATP.

\section{Piezo1 activation enhances calcium release from intracellular stores in}

\section{LPS-stimulated astrocytes}

To determine the effects of Piezo1 activation on $\mathrm{Ca}^{2+}$ release from internal stores, extracellular $\mathrm{Ca}^{2+}$ and magnesium $\left(\mathrm{Mg}^{2+}\right)$ were removed from the medium, i.e. HBSS minus $\mathrm{Ca}^{2+}$ and $\mathrm{Mg}^{2+}\left(\mathrm{HBSS}^{-/-}\right)$. Experiments were repeated, as above, with the same four groups of astrocytes, i.e. Control/HBSS ${ }^{-/-}$, Control/Yoda1 ${ }^{-/}$, LPS/HBSS $^{-/-}$and LPS/Yoda1 ${ }^{-/-}$(Fig. 4A - C). Interestingly, Yoda1 ${ }^{-/-}$(dissolved in HBSS minus $\mathrm{Ca}^{2+}$ and $\mathrm{Mg}^{2+}$ ) caused a large efflux of $\mathrm{Ca}^{2+}$ from control astrocytes; -63.8 a.f.u (-98.6, -7.3 IQR), but LPS-stimulated astrocytes showed a net increase in cytosolic $\mathrm{Ca}^{2+}$ levels; +11.0 a.f.u (-50.6, +75.8 IQR) (Fig. 5A). As such, Yoda1 increased the proportion of cells in non-treated astrocytes that displayed zero positive peak deflections in intracellular $\mathrm{Ca}^{2+}$ (i.e. Control/Yoda1 ${ }^{-/-} 55 \%$ vs $34 \%$ Control/HBSS ${ }^{-/}, \chi^{2}$ test, $\left.\mathrm{p}<0.001\right)$. This was due to the large net efflux of $\mathrm{Ca}^{2+}$ to the extracellular medium and, therefore, the decrease in basal $\mathrm{Ca}^{2+}$ levels in the Control/Yoda1 ${ }^{-/}$group. However, comparable proportions of cells $(24 \%)$ in the Control/Yoda1 ${ }^{-/}$group responded with one positive $\mathrm{Ca}^{2+}$ peak compared to astrocytes in the Control/HBSS ${ }^{-/-}$group (27\%). Therefore, the difference lay in the proportion of cells that displayed $\mathrm{Ca}^{2+}$ oscillations (i.e. 2 or more peaks). $21 \%$ of Control/Yoda1 ${ }^{-/-}$cells displayed $\mathrm{Ca}^{2+}$ oscillations in response to Yoda1 whereas $39 \%$ of cells in the Control/HBSS ${ }^{-/-}$group showed $\mathrm{Ca}^{2+}$ oscillations following 
$\mathrm{HBSS}^{-/-}$perfusion (Fig. 5B). This indicates that Yoda ${ }^{-/-}$decreases $\mathrm{Ca}^{2+}$ oscillations driven by internal stores in control non-reactive astrocytes $\left(\chi^{2}\right.$ test, $\left.\mathrm{p}<0.001\right)$.

The opposite was true for the LPS/HBSS ${ }^{-/}$group, however. A similar proportion of non-responders $(32 \%)$ were measured in $\mathrm{LPS} \mathrm{HBSS}^{-/-}$treated astrocytes compared to $34 \%$ of cells in the Control/ $\mathrm{HBSS}^{-/-}$group. However, there was a $10 \%$ increase in cells displaying $\mathrm{Ca}^{2+}$ oscillations ( $\mathrm{LPS} / \mathrm{HBSS}^{-/} 49 \%$ vs $39 \%$ Control/HBSS ${ }^{-/}, \chi^{2}$ test, $\mathrm{p}<0.001$ ), suggesting that LPS-stimulated astrocytes are more 'responsive' to shear stress and display more internal store-driven $\mathrm{Ca}^{2+}$ oscillations. Interestingly, Yodal induced a modest $4 \%$ increase in the proportion of cells that displayed $\mathrm{Ca}^{2+}$ oscillations in LPS-stimulated cultures (LPS/HBSS ${ }^{-/} 49 \%$ vs $53 \%$ LPS/Yoda1 ${ }^{-/-}, \chi^{2}$ test, $\mathrm{p}=0.008)$. However, Yodal caused a greater increase in the proportion of cells that respond with just one peak in intracellular $\mathrm{Ca}^{2+}\left(\mathrm{LPS} / \mathrm{HBSS}^{-/-} 19 \%\right.$ vs $28 \%$ LPS/Yoda1 ${ }^{-/}$) (Fig. 5B). This increase in internal store-driven $\mathrm{Ca}^{2+}$ oscillations in LPS-treated astrocytes exposed to Yodal impacted their subsequent responses to ATP perfusion. As such, $68 \%$ of astrocytes in the LPS/Yoda1 ${ }^{-/}$group responded to ATP with one positive peak in intracellular $\mathrm{Ca}^{2+}$ versus $61 \%$ of $\mathrm{LPS}^{2} \mathrm{HBSS}^{-/-}$ astrocytes $\left(\chi^{2}\right.$ test, $\left.\mathrm{p}<0.001\right)$. However, this meant that $39 \%$ of $\mathrm{LPS} / \mathrm{HBSS}^{-/-}$ astrocytes showed ATP-induced $\mathrm{Ca}^{2+}$ oscillations versus $32 \%$ of the LPS/Yoda1 ${ }^{-/}$ group. The proportion of cells showing $\mathrm{Ca}^{2+}$ oscillations to ATP in the Control/Yoda1 ${ }^{-/}$group was even lower again (24\%) compared to the Control/HBSS ${ }^{-/}$group (33\%). Therefore, whilst Yodal caused an increase in ATPinduced $\mathrm{Ca}^{2+}$ release from intracellular stores in control astrocytes (Fig. 5C) and increases the percentage of cells that respond with one $\mathrm{Ca}^{2+}$ peak following ATP perfusion (Fig. 5D), astrocyte cultures in the Control/Yoda1 ${ }^{-/-}$group displayed the lowest proportion of cells (24\%) that oscillate in response to Yoda1 perfusion (Fig. 5D). Taken together, LPS-stimulated astrocytes respond to Yoda1 by increasing intracellular $\mathrm{Ca}^{2+}$ levels and internal store-driven $\mathrm{Ca}^{2+}$ oscillations compared to astrocytes perfused with control $\mathrm{HBSS}^{-/}$. However, LPS/Yoda1 ${ }^{-/-}$astrocytes were less responsive to ATP, presumably because their basal $\mathrm{Ca}^{2+}$ levels were higher than the Control/Yoda1 ${ }^{-/}$group. 


\section{Blocking Piezo1 channels enhances cell migration in LPS-stimulated astrocytes}

Astrocytes were treated with LPS $(100 \mathrm{ng} / \mathrm{mL})$ for $48 \mathrm{~h}$ in the presence or absence of either Yoda1 (agonist) or GsMTx4 (antagonist) to activate or inhibit Piezo1 channels, respectively. Neither activation nor inhibition of Piezol affected cell viability (Fig. 6A) or mitochondrial stress (Fig. 6B). Because Piezo1 channels have been shown previously to regulate endothelial (Eisenhoffer et al., 2012), fibroblast (Chubinskiy-Nadezhdin et al., 2019) and cancer cell migration (McHugh, Murdoch, Haslett, \& Sethi, 2012), we next investigated if Piezo1 channels modulate astrocyte migration. Control astrocytes were grown to confluency over a $500 \mu \mathrm{m}$ silicone strip running down the centre of a $35 \mathrm{~mm}$ cell culture well. The silicone strip was then peeled away to create a reproducible 'wound healing' type assay to measure collective astrocyte migration velocities over a 48-hour period. Astrocytes were exposed to either Yoda1 $(10 \mu \mathrm{M})$ or GsMTx4 $(500 \mathrm{nM})$ for $48 \mathrm{~h}$, neither of which had any significant effect on astrocyte migration compared to non-treated control cells which migrated at an average velocity of $3.3 \pm 1.0 \mu \mathrm{m} / \mathrm{h}$ (Fig. 6C). Astrocytes treated with GsMTx 4 migrated at $3.8 \pm 0.6 \mu \mathrm{m} / \mathrm{h}$ but this was not statistically significantly faster than astrocytes treated with Yoda1 $(2.0 \pm 0.4 \mu \mathrm{m} / \mathrm{h})$. In order to assess the role of Piezo1 in cell migration, astrocytes were stimulated with LPS to trigger Piezo 1 expression and migratory velocities were monitored over $48 \mathrm{~h}$. LPSstimulated astrocytes exposed to GsMTx 4 for $48 \mathrm{~h}$ migrated at a velocity of $5.0 \pm$ $0.9 \mu \mathrm{m} / \mathrm{h}$. This was not significantly faster than astrocytes treated with LPS alone $(2.3 \pm 0.3 \mu \mathrm{m} / \mathrm{h})$ but was significantly faster than LPS-stimulated astrocytes exposed to Yoda1 $(1.3 \pm 0.5 \mu \mathrm{m} / \mathrm{h})($ Fig. $6 \mathrm{C})$. This suggests that Piezol channel activity regulates astrocyte migration under neuroinflammatory conditions (Fig. $6 \mathrm{D}, \mathrm{E})$.

Yoda1 inhibits cytokine and chemokine release from LPS-stimulated astrocytes

LPS is a well-known trigger of inflammation and cytokine release from astrocytes (Liddelow et al., 2017). Our next aim was to investigate if modulating Piezo1 activity alters pro-inflammatory cytokine or chemokine release from cortical 
astrocyte cultures. Treating astrocytes with Yoda1 for $48 \mathrm{~h}$, in the presence of LPS, decreased the release of TNF $\alpha(484 \pm 157 \mathrm{vs} 2,046 \pm 410 \mathrm{pg} / \mathrm{mL})$ (Fig. 7A), IL-1 $\beta$ $(111 \pm 12$ vs $515 \pm 122 \mathrm{pg} / \mathrm{mL})$ (Fig. 7B), and CX ${ }_{3} \mathrm{CL} 1(4,089 \pm 430$ vs 11,745 \pm $1,210 \mathrm{pg} / \mathrm{mL}$ ) (Fig. 7D), but had no significant effect on IL-6 release $(4,274 \pm 601$ vs $6,468 \pm 1,202 \mathrm{pg} / \mathrm{mL}$ ) (Fig. 7C). However, there was a statistically significant difference in the amount of IL-6 released from astrocytes treated with LPS/Yoda1 $(4,274 \pm 601 \mathrm{pg} / \mathrm{mL})$ versus LPS/GsMTx4 (8,076 $\pm 947 \mathrm{pg} / \mathrm{mL})$ (Fig. 7C). Interestingly, Yoda1 also decreased $\mathrm{CX}_{3} \mathrm{CL} 1$ release from control astrocytes $(3,105$ \pm 94 vs $10,140 \pm 741 \mathrm{pg} / \mathrm{mL})$. Taken together, our results suggest that LPS exposure upregulates Piezo1 in a modest proportion of astrocytes $(\sim 25 \%)$ and, when activated, Piezol channels increase intracellular $\mathrm{Ca}^{2+}$ oscillations and inhibit the production and release of pro-inflammatory cytokines and chemokines (Fig. 8). Astrocytic Piezol may, therefore, act to dampen CNS neuroinflammation. 


\section{Discussion}

Our understanding of glial cell biology and biophysics has advanced rapidly over the last two decades and it is now widely accepted that astrocytes resident in different brain regions exhibit heterogeneity in morphology and function (Matias, Morgado, \& Gomes, 2019). Local sub-populations of astrocytes within a common neuronal ensemble also appear to exhibit distinct functional groupings, such as the A1 and A2 phenotypes recently described (Liddelow et al., 2017). Astrocytes sense and react to the local biochemical milieu of the brain parenchyma, as well as to the mechanical properties of the extracellular matrix and neighbouring cells, particularly after CNS injury (Bowers, Fiori, Khadela, Janmey, \& Galie, 2019; Rocha, Ferraz-Nogueira, Barrias, Relvas, \& Pego, 2015). Here, primary mouse astrocyte cultures were used to investigate several potential functions of astroglial Piezo1 channels in the cerebral cortex during a neuroinflammatory stimulus. Our data suggest that the bacterial endotoxin, LPS, triggers Piezo1 expression in cortical astrocytes which normally express very low levels of mechanosensitive Piezo1 ion channels, at least under control conditions in vitro, although we and others have detected astrocytic Piezo1 expression in vivo (Choi, Sun, \& Jakobs, 2015; VelascoEstevez et al., 2018). However, not all LPS-stimulated astrocytes ( 25\%) expressed the Piezo1 protein in vitro, suggesting those that did may have slightly different functions to their Piezo1-negative neighbours. Alternatively, astrocytes in culture may display a range of responses to LPS stimulation. For instance, the levels of TLR4 receptor expression may vary between functionally distinct subpopulations of cultured astrocytes (El-Hage et al., 2011) which could also explain why only $\sim 25 \%$ of astrocytes expressed Piezo1 post-LPS treatment.

\section{Astrocyte migration}

Astrocyte migration was assessed using a two-dimensional wound healing-type assay and, therefore, resembled an unrestricted collective cell migration. Cell migration can be classified using several criteria, e.g. single cell vs collective migration (Rorth, 2009), ameboid vs integrin-dependant (Friedl \& Brocker, 2000) or confined vs unrestricted migration (Hung et al., 2016). In the healthy adult brain, 
astrocytes do not generally migrate (Zhan et al., 2017). However, in neuroinflammatory states or following traumatic tissue injury, reactive astrocytes can change shape, become hypertrophic (Sofroniew \& Vinters, 2010), contribute to glial scarring (Buffo, Rolando, \& Ceruti, 2010) and display integrin-dependent migration (Cardenas, Kong, Alvarez, Maldonado, \& Leyton, 2014). Interestingly, before its characterisation as a mechanosensitive ion channel, McHugh et al. (2010) described an important role for Piezol in maintaining integrin-mediated cell adhesion in epithelial cells. Piezo1 reportedly recruits the small GTPase, R-Ras, to the endoplasmic reticulum leading to release of calcium from internal stores, activation of calpain signalling, cleavage of talin and enhancement of integrin affinity, thus strengthening cell adhesions. Moreover, knocking down Piezo1 using siRNA increased migration of lung epithelial cells through loss of integrin-mediated cell adhesions (McHugh et al., 2012). Therefore, it has been suggested that Piezol expression may influence the metastatic invasiveness of cancer cells ( $\mathrm{Li}$ et al., 2015; Yang et al., 2014). However, Piezo1 antagonism appears to have different effects on migration in different cell types. Treating human umbilical vein endothelial cells (HUVECs) or prostate cancer cell lines (PC-3 and LNCaP) with GsMTx4 causes a decrease in cell migration (Li et al., 2014; Maroto, Kurosky, \& Hamill, 2012) but enhances neurite outgrowth in Xenopus spinal cord explants (Jacques-Fricke, Seow, Gottlieb, Sachs, \& Gomez, 2006). More recently, Song and colleagues have shown that Piezo1 activation can inhibit neural regeneration in Drosophila (Song et al., 2019). Here, we show that astrocytes exposed to LPS (to induce Piezo1 expression) and GsMTx4 (to simultaneously block Piezo1 channel opening) migrate faster than astrocytes stimulated with LPS and Yoda1 (which activates Piezo1 channels). It is possible that GsMTx4 modulates integrin affinity in reactive astrocytes, thus enhancing collective cell migration velocity. In agreement with our findings, Chubinskiy-Nadezhdin et al. (2019) have also recently reported that Yoda1 decreases fibroblast migratory speed. Therefore, we hypothesise that activation of Piezo1 may attenuate the reactive phenotype of astrocytes in vivo by dampening their migratory capacity in neuroinflammatory disorders of the ageing brain, for example. 


\section{Piezo1-mediated $\mathrm{Ca}^{2+}$ signalling in astrocytes}

Under non-inflammatory control conditions, cultured astrocytes appeared largely devoid of Piezo1 expression; although approximately $48 \%$ of cells responded to Yoda1 application with small influxes of calcium (Fig. 3A, B). Therefore, Piezo1 channels may be expressed at low levels which are undetectable with the antibody used here. As a positive modulator of the Piezo1 ion channel, Yoda1 binds directly to Piezo1 to stabilize the open conformation of the channel pore, thus decreasing the threshold for mechanical activation (Cahalan et al., 2015; Syeda et al., 2015; Lacroix et al., 2018). However, Yoda1 may also exert some off-target effects, such as increasing the likelihood that other types of $\mathrm{Ca}^{2+}$-permeable channels will open, as suggested by Dela Paz \& Frangos (2018) who showed that Yoda1 activates both Akt and ERK1/2 signalling in endothelial cell types, independent of Piezo1. The authors argue that Yoda1 may activate one or more transient receptor potential (TRP) cation channels which could explain how Akt and ERK1/2 become phosphorylated. This could also explain why, in our study, $\sim 48 \%$ of control astrocytes responded to Yoda1 with small influxes of $\mathrm{Ca}^{2+}$, but we could detect only $\sim 3 \%$ of cells that expressed Piezo1 (as quantified by immunofluorescence). Following $24 \mathrm{~h}$ stimulation with LPS, however, Piezo1 was upregulated in $~ 25 \%$ of astrocytes and much larger $\mathrm{Ca}^{2+}$ influxes were observed (Fig. 3A). Moreover, $60 \%$ of reactive astrocytes responded to Yoda1 versus $48 \%$ of control astrocytes (Fig. 3B). Therefore, the predominant component of the Yoda1-mediated $\mathrm{Ca}^{2+}$ response in reactive astrocytes is likely regulated by Piezo1 channels, even though a smaller off-target component is also detectable.

Our data also demonstrate that Piezo1-mediated $\mathrm{Ca}^{2+}$ influx into LPS-stimulated astrocytes may trigger internal store-driven $\mathrm{Ca}^{2+}$ oscillations. In the absence of extracellular $\mathrm{Ca}^{2+}$, Yoda1 induced a net efflux of $\mathrm{Ca}^{2+}$ to the extracellular space of control astrocytes. In contrast, Yodal caused a net enhancement of cytosolic $\mathrm{Ca}^{2+}$ concentration in LPS-reactive astrocytes (Fig. 5A). Since Piezo1 expression is almost absent in control astrocytes, Yoda1-mediated efflux of $\mathrm{Ca}^{2+}$ may be caused by the non-specific opening of membrane-bound cation channels (e.g. TRP channels), thus allowing $\mathrm{Ca}^{2+}$ to flow out of the cell and into the $\mathrm{Ca}^{2+}$ and $\mathrm{Mg}^{2+}$ free 
medium. LPS-reactive astrocytes, on the other hand, upregulate Piezol channels (Fig. 1) and given that Piezo1 can localise to the ER (Mc Hugh et al., 2010), our data suggests that Yoda1 triggers calcium release from internal stores, possibly via intracellular Piezo1 channels (Fig. 8). Moreover, depletion of $\mathrm{Ca}^{2+}$ from internal stores can activate calcium release-activated channels (CRAC) in the outer membrane, thus enhancing extracellular $\mathrm{Ca}^{2+}$ influx and facilitating the replenishment of internal calcium stores (Prakriya, 2009). Indeed, in the presence of extracellular $\mathrm{Ca}^{2+}$, we found that Yoda1 initially enhances cytosolic $\mathrm{Ca}^{2+}$ levels (most likely via $\mathrm{Ca}^{2+}$ influx through membrane-bound Piezo1 channels), which in turn has the potential to cause calcium-induced calcium release (CICR) from intracellular stores. CICR can also trigger further $\mathrm{Ca}^{2+}$ entry via membraneexpressed store-operated calcium channels (SOCs), such as TRP cation channels, which are expressed both on the outer cell membrane as well as localised to the ER (Dong et al., 2010; Verkhratsky \& Parpura, 2014). Taken together, this could explain why we observed more $\mathrm{Ca}^{2+}$ influx $2-4$ min post-Yoda1 exposure versus the first 2 min of Yoda1 treatment (Fig. 3A).

\section{Piezo1 and ATP-responsiveness in astrocytes}

Prior activation of Piezo1, with Yoda1, decreased $\mathrm{Ca}^{2+}$ influx in response to ATP stimulation. This suggests that Piezo1 activation in reactive astrocytes could have both local and long-range consequences for neurotransmission in the cerebral cortex. Indeed, paracrine signalling in astrocyte networks is facilitated through ATP-mediated upregulation of inositol trisphosphate $\left(\mathrm{IP}_{3}\right)$ which triggers the release of $\mathrm{Ca}^{2+}$ from internal stores (Fumagalli et al., 2003; Kastritsis, Salm, \& McCarthy, 1992), and this, in turn, causes the release of more ATP from the astrocyte. ATP released from astrocytes can activate purinergic (P2X) receptors on the post-synaptic neuron and induce metaplastic changes in synaptic strength (Boue-Grabot \& Pankratov, 2017). Bi-directional modulation of glutamatergic synaptic transmission has been demonstrated through either slow and prolonged decreases in AMPA receptor-mediated mini excitatory post-synaptic currents (mEPSCs) (Pougnet et al., 2014) or via P2X-mediated insertion of AMPA receptors 
into the post-synaptic membrane which culminates in the long-term enhancement of mEPSC amplitude (Gordon et al., 2005).

ATP is also important in the propagation of $\mathrm{Ca}^{2+}$ waves through astrocyte networks (Fujii, Maekawa, \& Morita, 2017). Moreover, vasoactive substance release is regulated by astrocytic $\mathrm{Ca}^{2+}$ waves and ATP-induced $\mathrm{Ca}^{2+}$ release from intracellular stores within astrocytes (Metea \& Newman, 2006). Therefore, astrocyte branches release factors that can induce vasoconstriction or vasodilation of cerebral vasculature (Filosa \& Iddings, 2013). Piezo1-mediated dampening of ATP-induced $\mathrm{Ca}^{2+}$ waves in astrocytes could, in theory, regulate cerebral blood flow. Indeed, Piezo1 is activated by shear stress which leads to increases in nitric oxide synthase and NO release from vasculature-derived endothelial cells (Wang et al., 2016). Since astrocytic endfeet are in contact with pericytes that wrap around cerebral blood vessels (Abbott, Ronnback, \& Hansson, 2006; Bonkowski, Katyshev, Balabanov, Borisov, \& Dore-Duffy, 2011), it would be interesting to study the role of astrocytic Piezol in the regulation of vascular tone and blood brain barrier permeability in future experiments.

\section{Piezo1 and neuroinflammation}

Because astrocytes are also an important source of neuromodulatory cytokine and chemokine production post-LPS exposure (Lieberman, Pitha, Shin, \& Shin, 1989; Rothhammer \& Quintana, 2015), we measured the effects of Piezo1 activation on IL-1 $\beta, T N F \alpha$, IL-6 and $\mathrm{CX}_{3} \mathrm{CL} 1$ release from reactive astrocytes. Blocking Piezo1 channels, using GsMTx4, had little or no effect on cytokine release. However, activating Piezo1 channels with Yoda1 significantly decreased pro-inflammatory cytokine release from LPS-stimulated cortical astrocytes. We also found that Yoda1 decreased $\mathrm{CX}_{3} \mathrm{CL} 1$ release from unstimulated control astrocytes (Fig. 7D). Therefore, the anti-inflammatory effects of Yoda1 may not be entirely dependent on Piezo1 activation. Yoda1 may cause a general dampening of cytokine secretory activity in astrocytes. 
Although LPS-induced up-regulation of Piezo1 in cultured astrocytes is somewhat transient (Fig. 1), with protein levels decreasing again after $48 \mathrm{~h}$; chronic overexpression of Piezo1 does occur in vivo, particularly in reactive astrocytes surrounding amyloid plaques in the ageing brain of Alzheimer's disease (AD) rats harbouring a chronic urinary tract infection (Velasco-Estevez et al., 2018). It is interesting to speculate on the potential functional roles of Piezo1, as well as the potential triggers of mechanosensitive channel opening, in the ageing and neurodegenerating brain (Tyler, 2012). We hypothesise that stiff amyloid plaques scattered randomly throughout the $\mathrm{AD}$ brain act as extrinsic mechanical modulators of Piezo1 channel opening probability. In addition, Piezo1 activity may be modulated by the gradual softening of ageing AD brain tissue (ElSheikh et al., 2017) which also undergoes extracellular matrix (ECM) remodelling at sub-cellular length scales (Sethi \& Zaia, 2017). Moreover, because amyloid plaques represent an altered extrinsic mechanical environment for astrocytes in vivo, Piezo1 ion channels could potentially modulate mechanotransduction signalling in reactive astrocytes in response to amyloid accumulation. Interestingly, Piezo1 regulates the expression of the mechanoresponsive transcriptional cofactor Yes-associated protein (YAP) and activates the Hippo signalling pathway (Pathak et al., 2014) which controls important processes such as cell contact inhibition and cell proliferation (Pavel et al., 2018). YAP is highly expressed by astrocytes and can activate suppressor of cytokine signalling (SOCS), thus preventing astrocyte reactivity by downregulating the JAK-STAT inflammatory pathway (Huang et al., 2016). It will be important in future experiments to investigate the potential antiinflammatory mechanism of action of astrocytic Piezo1 in more detail and to determine if Piezo1 could be a novel drug target for neuroinflammatory disorders of the ageing brain. 


\section{Conclusion}

The data presented here suggests that mechanosensitive astrocytes upregulate Piezo1 channels in response to LPS in an attempt to inhibit pro-inflammatory cytokine release and dampen neuroinflammation. Activation of Piezol channels with Yoda1 slowed astrocyte migration and blocked TNF $\alpha$, IL-1 $1 \beta$ and $\mathrm{CX}_{3} \mathrm{CL} 1$ release from LPS-stimulated astrocytes. This is likely through $\mathrm{Ca}^{2+}$-dependent signalling cascades since Piezol activation enhanced both extracellular $\mathrm{Ca}^{2+}$ influx and internal store-mediated release of $\mathrm{Ca}^{2+}$ into the cytosol of reactive astrocytes. Moreover, Piezo1-mediated $\mathrm{Ca}^{2+}$ influx dampens subsequent ATP-induced $\mathrm{Ca}^{2+}$ responses in astrocytes, suggesting that Piezol could modulate neurotransmission by regulating gliotransmitter release at the synapse. It will be important, going forward, to investigate if there is a link between $\mathrm{Ca}^{2+}$-mediated mechanotransduction in astrocytes and other well-known biochemical signalling pathways that regulate synaptic plasticity, particularly in the ageing neuroinflammatory brain. 


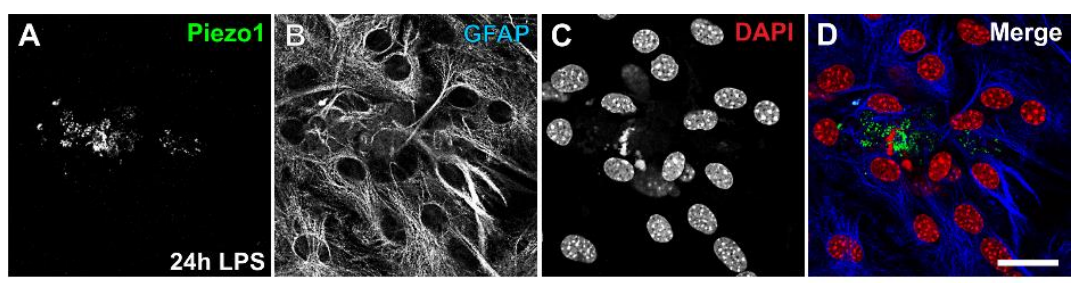

E

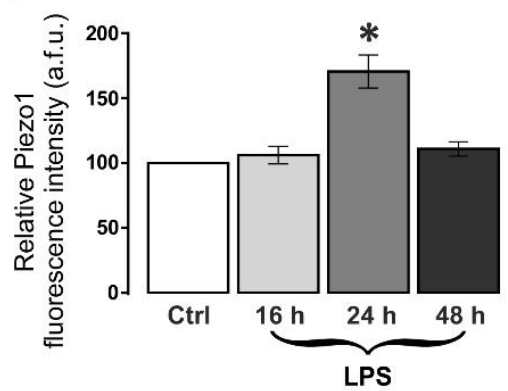

G

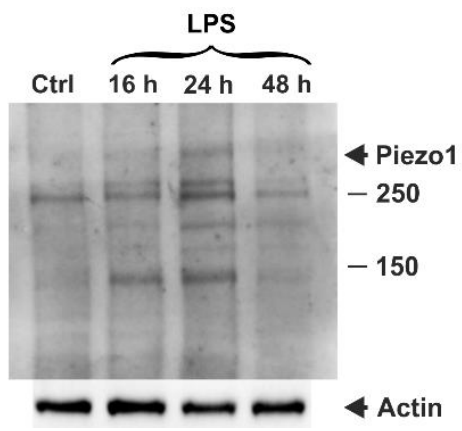

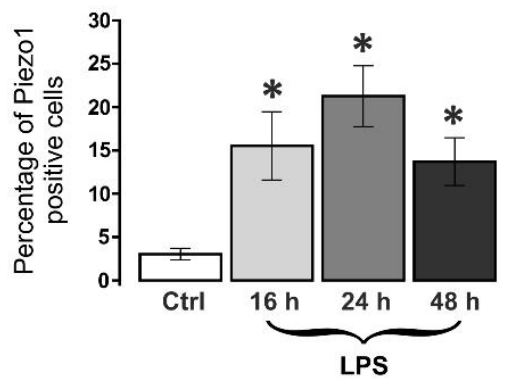

H

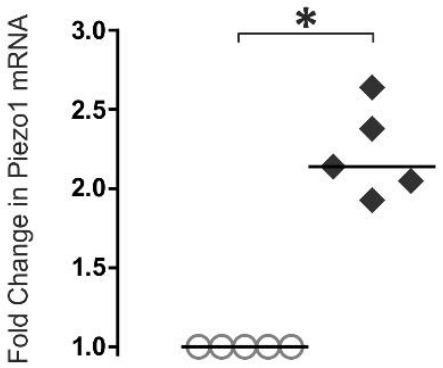

Figure 1: Lipopolysaccharide induces Piezo1 expression in astrocytes. Mouse cortical astrocytes were treated with $100 \mathrm{ng} / \mathrm{mL}$ LPS for 16,24 or $48 \mathrm{~h}$ and then immunofluorescently labelled for (A) Piezo1 channels, (B) the astrocytic marker GFAP, and (C) nuclei were counterstained with DAPI. (D) Image represents the above three channels merged. Scale bar $=$ $20 \mu \mathrm{m}$. (E) LPS caused an upregulation of Piezo1 in astrocytes after $24 \mathrm{~h}$, measured as an increase in Piezo1 fluorescence intensity co-localised with GFAP. (F) Only $2-3 \%$ of astrocytes expressed detectable levels of Piezo1 in control non-treated cultures. However, this rose to $\sim 15 \%$ after $16 \mathrm{~h}$ LPS and peaked at $20-25 \%$ of Piezo1-positive astrocytes following $24 \mathrm{~h} \mathrm{LPS}$ exposure. Immunofluorescence experiments were repeated $n=5$ with two technical replicates per experiment. Results are displayed as mean \pm S.E.M and were analysed using a one-way ANOVA with Holm-Sidak post hoc test, ${ }^{*} \mathrm{p}<0.05$. (G) Results obtained through immunofluorescence were confirmed by Western blot $(n=3)$ where a detectable increase in Piezo1 protein ( $286 \mathrm{kDa}$ band) was observed in astrocytes after $24 \mathrm{~h}$ LPS exposure. (H) Upregulation of astrocytic Piezo1 24 h post-LPS exposure was further confirmed by PCR analysis of mRNA transcript levels. PCR experiments were repeated $n=5$ with two technical replicates per experiment. Results are displayed as fold change from control levels and were analysed using a paired Student's T test, ${ }^{*} \mathrm{p}<0.05$. 
A

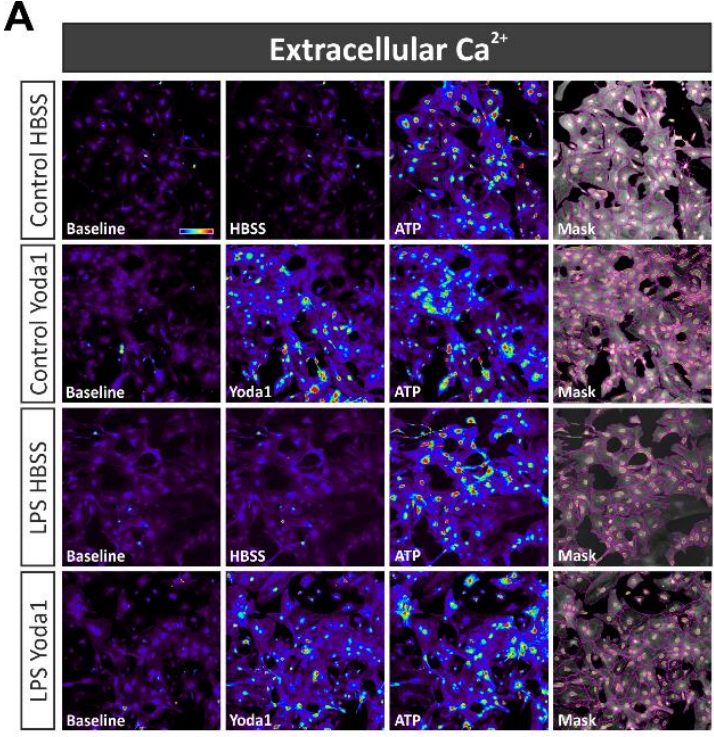

B

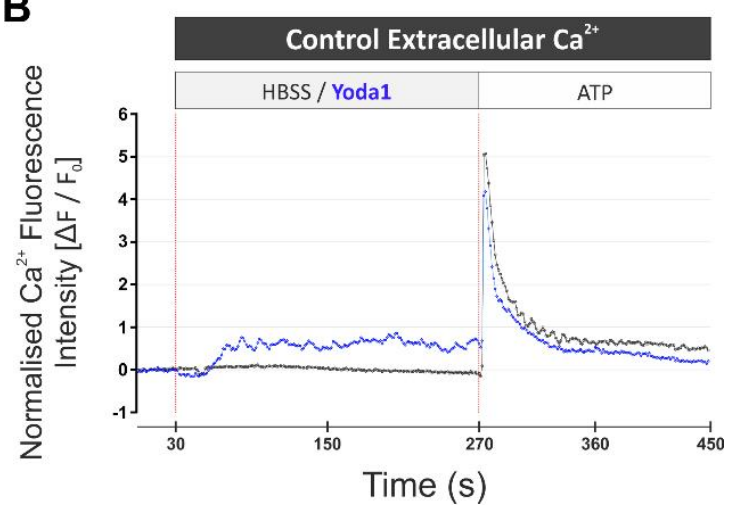

C

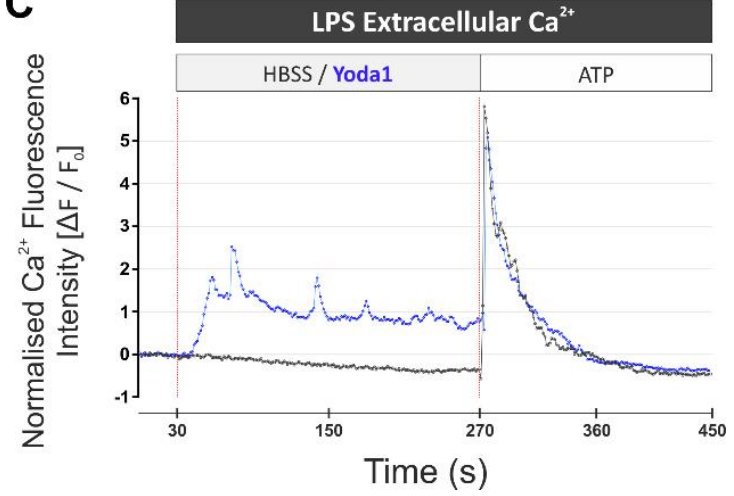

Figure 2: Activation of Piezo1 increases intracellular calcium in LPS-reactive astrocytes. Astrocytes were pre-treated with or without LPS (100 ng/mL) for $24 \mathrm{~h}$ and loaded with Cal-520AM calcium indicator for $2 \mathrm{~h}$ in Hank's balanced salt solution (HBSS) containing calcium $\left(\mathrm{Ca}^{2+}\right)$ and magnesium $\left(\mathrm{Mg}^{2+}\right)$. Immediately prior to $\mathrm{Ca}^{2+}$ imaging experiments, astrocytes were transferred to fresh HBSS with $\mathrm{Ca}^{2+}$ and $\mathrm{Mg}^{2+}$ (i.e. Extracellular $\mathrm{Ca}^{2+}$ group). (A) Representative confocal images (20x magnification) of baseline $\mathrm{Ca}^{2+}$ levels and $\mathrm{Ca}^{2+}$ influx in response to HBSS, Yoda1 and ATP in control and reactive astrocytes pre-treated with LPS for $24 \mathrm{~h}$. The rainbow-coloured palette represents intracellular $\mathrm{Ca}^{2+}$ concentrations with dark purple/black signifying low levels of $\mathrm{Ca}^{2+}$ and yellow/red representing high levels of $\mathrm{Ca}^{2+}$. Scale bar $=100 \mu \mathrm{m}$. The maximum intensity projections of all 349 images were used to segment astrocytes and delineate the nuclear and cytosolic compartments of each individual cell. All data are presented as changes in cytosolic $\mathrm{Ca}^{2+}$ influx as opposed to changes in nuclear $\mathrm{Ca}^{2+}$ concentrations. Time-lapse $\mathrm{Ca}^{2+}$ imaging experiments were performed at a rate of 0.77 frames per second over a $7 \min 30 \mathrm{~s}$ period (i.e. 349 images in total), which included $30 \mathrm{~s}$ baseline, 4 min exposure to $10 \mu \mathrm{M}$ Yoda1 or fresh HBSS, followed by stimulation with $50 \mu \mathrm{M}$ ATP for 3 min. (B) Representative traces showing changes in cytosolic $\mathrm{Ca}^{2+}$ levels in control and (C) LPS-stimulated astrocytes in response to HBSS (black) and Yoda1 (blue). There was a $30 \mathrm{~s}$ baseline, at which point HBSS or Yoda1 was perfused onto astrocyte cultures for 4 min followed by ATP stimulation (at 270 s) for a further $3 \mathrm{~min}$. Yoda1 caused larger elevations in cytosolic $\mathrm{Ca}^{2+}$ in LPS-treated astrocytes compared to control non-reactive astrocytes. 
A

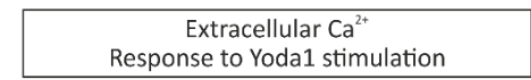

Response to Yoda1 stimulation
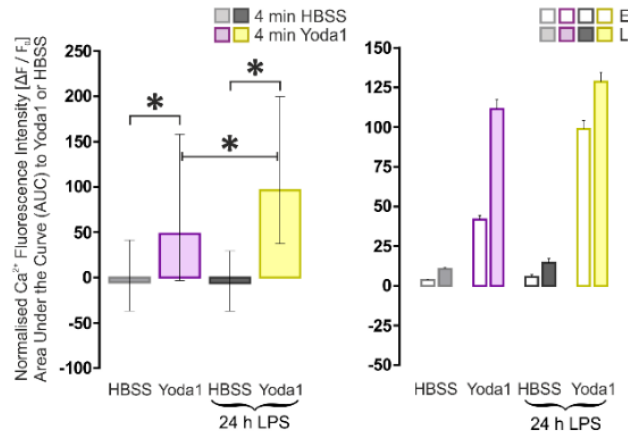

B

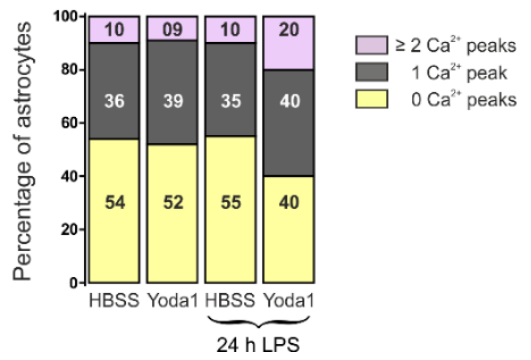

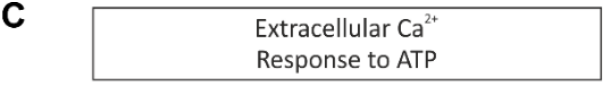
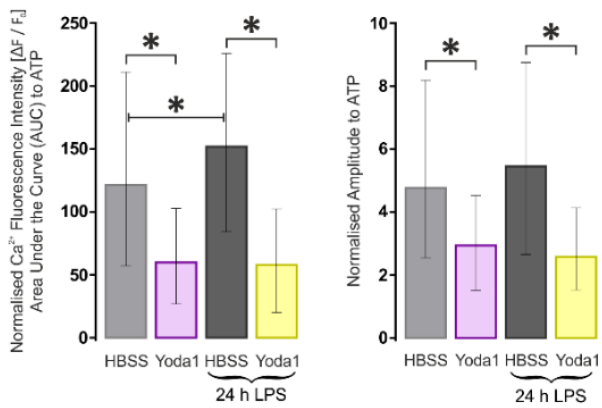

D

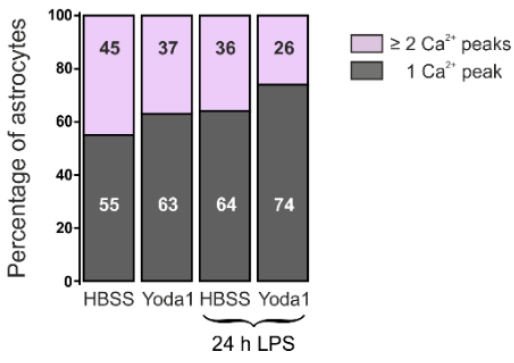

Figure 3: Activation of Piezo1 increases calcium oscillations in LPS-stimulated astrocytes.

(A) HBSS was used as a control for extracellular medium perfusion because Piezo1 channels are mechanosensitive and can be activated by shear stress. However, HBSS did not induce any appreciable increase in $\mathrm{Ca}^{2+}$ influx into control or LPS-stimulated astrocytes. Yoda1, on the contrary, caused an increase in $\mathrm{Ca}^{2+}$ influx into control astrocytes and a significantly larger influx of $\mathrm{Ca}^{2+}$ into LPS-stimulated astrocytes (+48.3 [-3.2, +158.1 IQR] vs +96.3 [37.5, 200.0 IQR] arbitrary fluorescence units; a.f.u). Data are presented as median \pm interquartile range and were analysed using a two-way ANOVA with Bonferroni post hoc test, $* \mathrm{p}<0.05$. The first 2 min of HBSS/Yoda1 exposure (designated "early") was also analysed separately to the last 2 min ("late"). In general, there was more $\mathrm{Ca}^{2+}$ influx in the latter 2 min period than the first 2 min, both in control and LPS-treated astrocytes, suggesting a delayed and prolonged increase in cellular $\mathrm{Ca}^{2+}$ levels in response to Yoda1 and, hence, Piezol channel activation. Early- and late-phase Yoda1 exposure data are presented as mean \pm the standard error of the mean (S.E.M). (B) Approximately $9-10 \%$ of control astrocytes stimulated with either HBSS or Yoda1, and LPS-stimulated astrocytes perfused with HBSS, displayed 2 or more $\mathrm{Ca}^{2+}$ peaks (i.e. $\mathrm{Ca}^{2+}$ oscillations). Interestingly, LPS-treated astrocytes stimulated with Yoda1 displayed double the number of cells expressing $\mathrm{Ca}^{2+}$ oscillations (i.e. $20 \%$ ), $\chi^{2}$ test, $\mathrm{p}<0.001$. This suggests that upregulation of Piezo1 in LPS-treated astrocytes may promote oscillatory $\mathrm{Ca}^{2+}$ activity in response to mechanical stimuli. (C) However, Yoda1-stimulated astrocytes responded less to ATP perfusion compared to HBSS-stimulated cells. Both the amplitude and the area under the 
curve (AUC) of the $\mathrm{Ca}^{2+}$ response to ATP was significantly reduced in Yoda1-stimulated astrocytes. Data are presented as median \pm interquartile range and were analysed using a twoway ANOVA with Bonferroni post hoc test, ${ }^{*} \mathrm{p}<0.05$. Astrocytes pre-treated with LPS for 24 $\mathrm{h}$ showed the largest AUC and peak amplitude to ATP, suggesting reactive astrocytes are more responsive to purinergic receptor stimulation. (D) However, only $26 \%$ of reactive astrocytes stimulated with Yoda1 displayed $\mathrm{Ca}^{2+}$ oscillations to ATP, whereas $36-45 \%$ of cells in the other three groups displayed ATP-induced $\mathrm{Ca}^{2+}$ oscillations. Therefore, Yoda1-mediated activation of Piezo1 appeared to dampen ATP-induced $\mathrm{Ca}^{2+}$ oscillations in astrocytes, $\chi^{2}$ test, $\mathrm{p}<0.001$. 
A

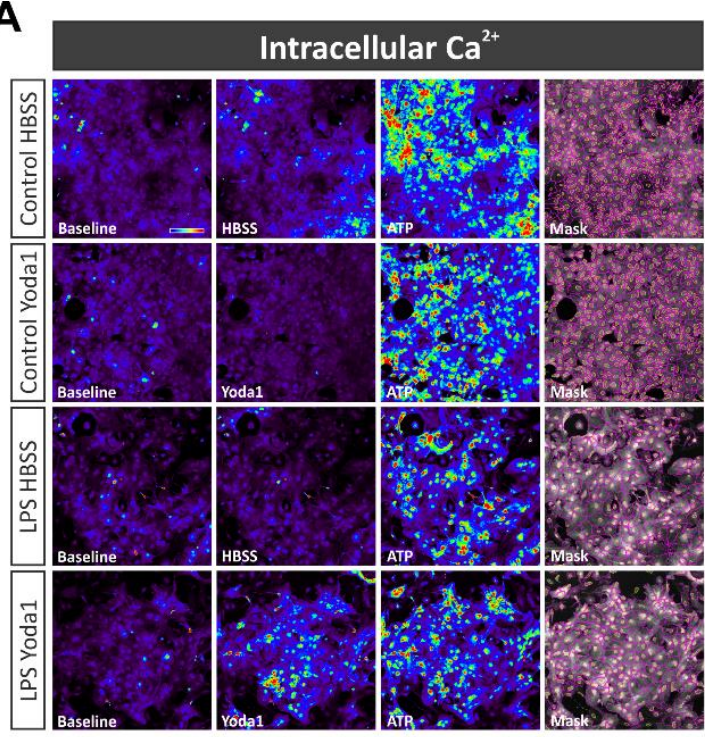

B

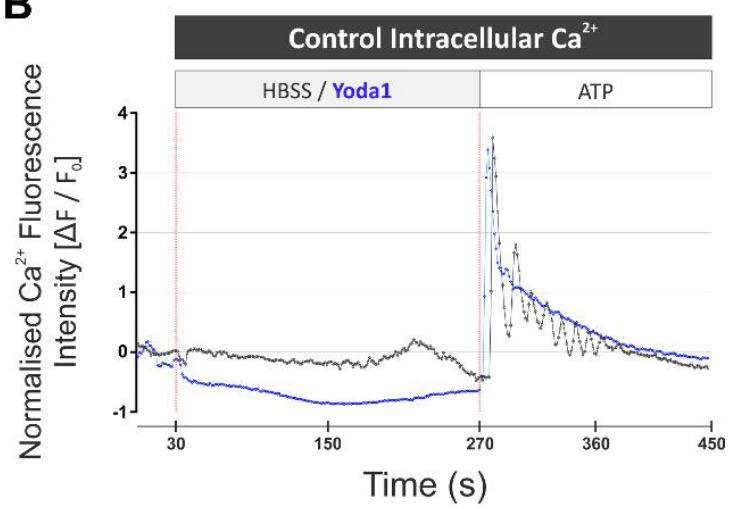

C

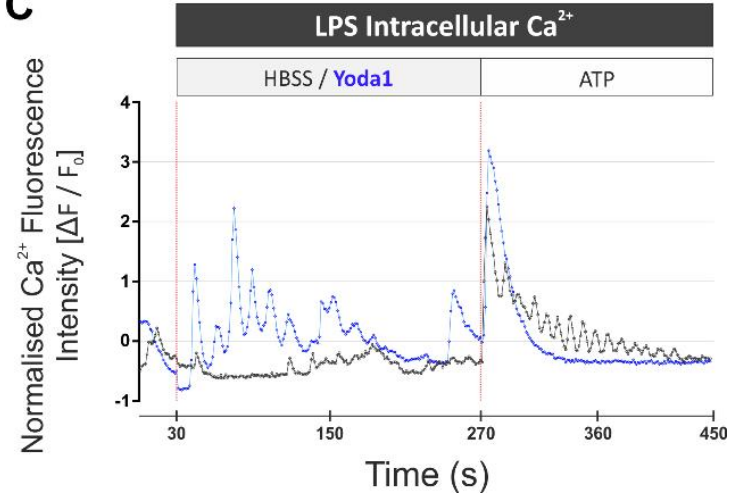

Figure 4: Activation of Piezo1 increases calcium release from intracellular stores in LPS-reactive astrocytes. Time-lapse $\mathrm{Ca}^{2+}$ imaging experiments were repeated in astrocyte cultures in the absence of extracellular calcium and magnesium (i.e. HBSS minus $\mathrm{Ca}^{2+}$ and $\mathrm{Mg}^{2+}$ ) in order to assess intracellular store-driven $\mathrm{Ca}^{2+}$ fluxes regulated by Piezo1 channels. Astrocytes were pre-treated with or without LPS (100 $\mathrm{ng} / \mathrm{mL}$ ) for $24 \mathrm{~h}$ and loaded with Cal520AM calcium indicator for $2 \mathrm{~h}$ in Hank's balanced salt solution (HBSS) containing calcium $\left(\mathrm{Ca}^{2+}\right)$ and magnesium $\left(\mathrm{Mg}^{2+}\right)$. Immediately prior to $\mathrm{Ca}^{2+}$ imaging experiments, astrocytes were transferred to fresh $\mathrm{HBSS}^{-/-}$without $\mathrm{Ca}^{2+}$ and $\mathrm{Mg}^{2+}$ (i.e. Intracellular $\mathrm{Ca}^{2+}$ group). Representative confocal fluorescence images of astrocytes loaded with Cal520AM dye and stimulated with $\mathrm{HBSS}^{--}$, Yoda1 $^{-/}$and $\mathrm{ATP}^{-/}$. The rainbow-coloured palette describes low versus high levels of intracellular $\mathrm{Ca}^{2+}$ concentrations. Scale bar $=100 \mu \mathrm{m}$. (B) Representative traces of control and (C) LPS-treated astrocytes stimulated with $\mathrm{HBSS}^{-/-}$or Yoda1 $^{-/-}$and $\mathrm{ATP}^{-/-}$in the absence of extracellular $\mathrm{Ca}^{2+}$ and $\mathrm{Mg}^{2+}$. Yoda1 caused a decrease in intracellular $\mathrm{Ca}^{2+}$ levels in control astrocytes, possibly through depletion of intracellular stores and efflux of $\mathrm{Ca}^{2+}$ from the cytosol. However, Yoda $1^{-/}$caused an increase in internal store-mediated $\mathrm{Ca}^{2+}$ elevations in LPS reactive astrocytes. 
A

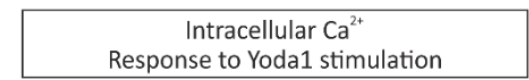

Response to Yoda1 stimulation
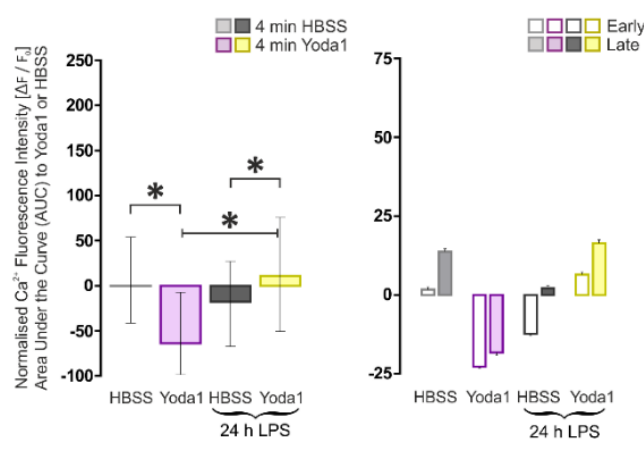

B

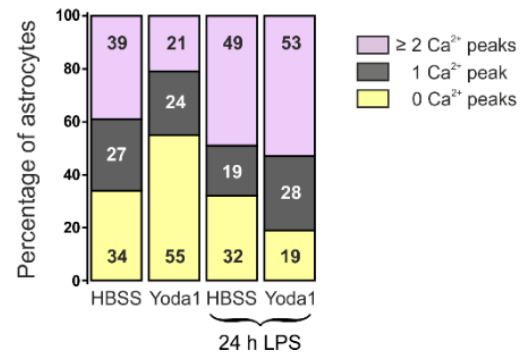

C
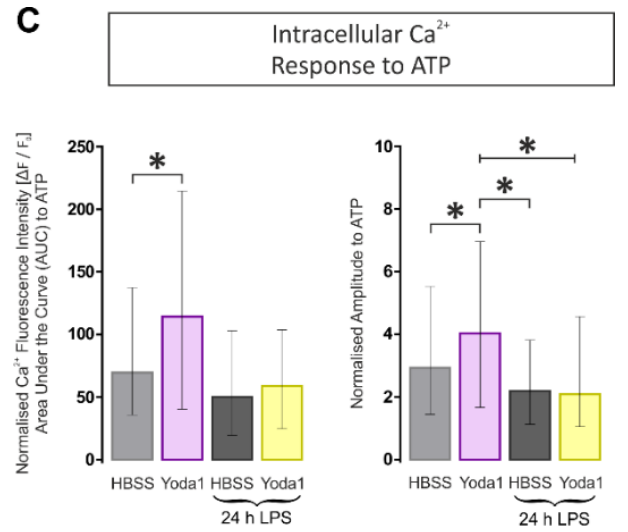

D

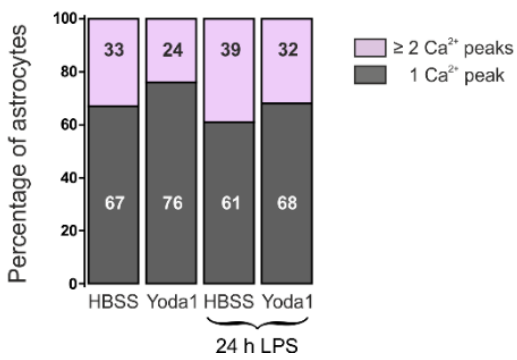

Figure 5: Yoda1 decreases ATP-induced calcium oscillations in astrocytes. (A) In control astrocytes, Yoda1 caused an efflux of $\mathrm{Ca}^{2+}$ from the cytosol to the extracellular space resulting in a decrease in Cal-520AM fluorescence intensity. In reactive astrocytes pre-treated with LPS for $24 \mathrm{~h}$, however, Yoda1 caused increases in cytosolic $\mathrm{Ca}^{2+}$ levels, presumably through release from intracellular stores but with a reduced efflux of $\mathrm{Ca}^{2+}$ ions to the extracellular space. Data for the total $4 \mathrm{~min}$ treatment period are represented as the median \pm the interquartile range and were analysed using a two-way ANOVA with Bonferroni post hoc test, * $\mathrm{p}<0.05$. The "early" and "late" phase data represent the mean \pm S.E.M. (B) Control astrocytes bathed in HBSS minus $\mathrm{Ca}^{2+}$ and $\mathrm{Mg}^{2+}$ and stimulated with Yoda1 displayed the lowest proportion of cells responding with multiple $\mathrm{Ca}^{2+}$ oscillations (21\%). This was due to the large efflux of $\mathrm{Ca}^{2+}$ in response to Yoda1. However, reactive astrocytes stimulated with Yoda1 displayed the largest proportion of intracellular store-driven $\mathrm{Ca}^{2+}$ oscillations (53\%), $\chi^{2}$ test, $\mathrm{p}=0.008$. (C) Interestingly, control astrocytes bathed in HBSS minus $\mathrm{Ca}^{2+}$ and $\mathrm{Mg}^{2+}$ and stimulated with Yoda1 showed the highest $\mathrm{Ca}^{2+}$ response to ATP, both in peak amplitude and AUC. This is likely due to the very low levels of cytosolic $\mathrm{Ca}^{2+}$ post-Yodal stimulation, i.e. the relative increases in $\mathrm{Ca}^{2+}$ appear very high when the basal levels are low. (D) Moreover, Yoda1 decreased the percentage of control cells that displayed ATP-induced store-driven $\mathrm{Ca}^{2+}$ oscillations (24\%), $\chi^{2}$ test, $\mathrm{p}<0.001$, suggesting that when basal $\mathrm{Ca}^{2+}$ levels are low, purinergic receptor stimulation does not induce many store-driven $\mathrm{Ca}^{2+}$ oscillations. 
A

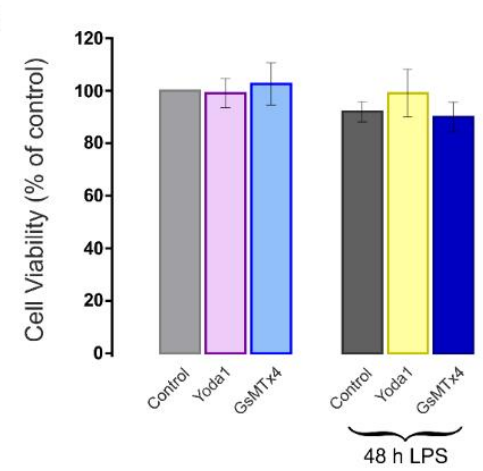

C

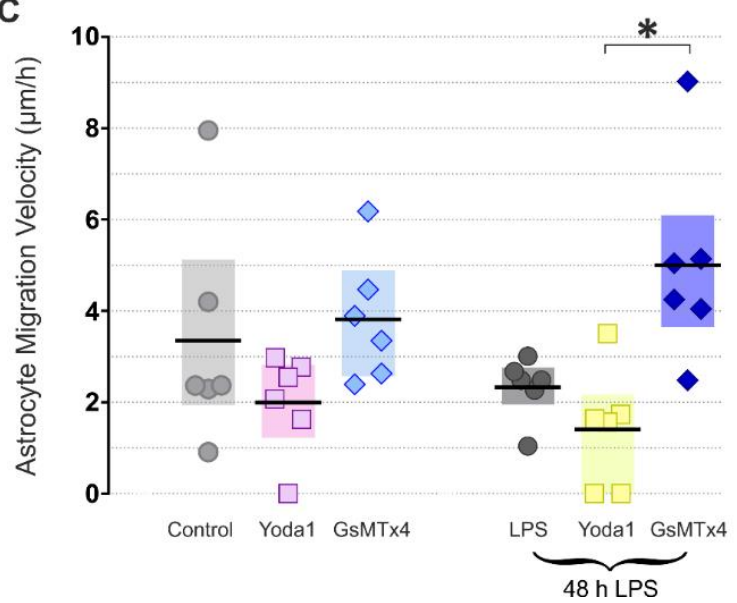

B
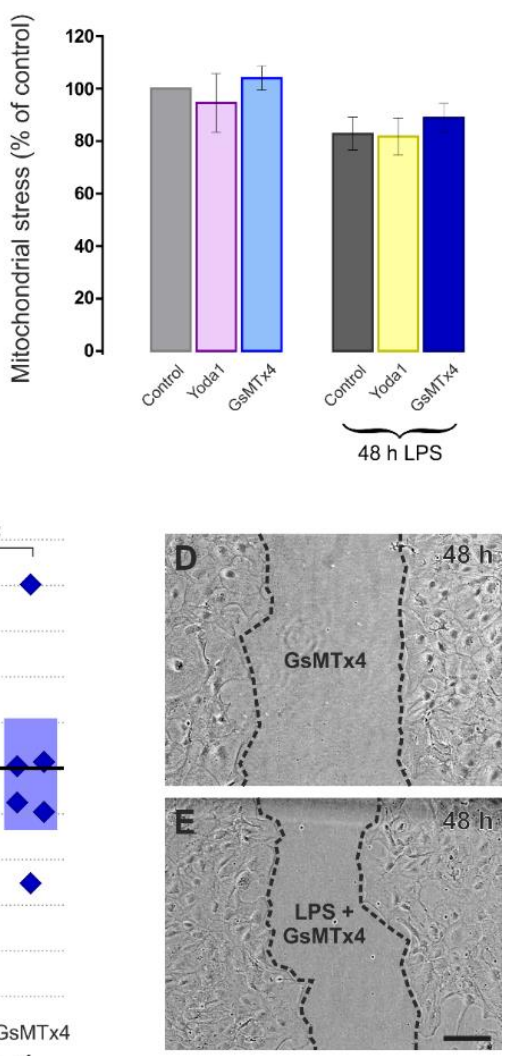

Figure 6: Piezo1 channels modulate astrocyte migration (A) Astrocyte cultures were treated with the Piezo1 agonist, Yoda1 $(10 \mu \mathrm{M})$, or the blocking peptide, GsMTx4 $(500 \mathrm{nM})$, in the presence or absence of LPS (100 ng/mL) for $48 \mathrm{~h}$. Neither Yoda1 nor GsMTx4 significantly affected cell viability of control or LPS-stimulated astrocytes as measured by the MTT assay. Data are presented as a percentage of the mean control fluorescence intensity $(540 / 590 \mathrm{~nm}) \pm$ S.E.M from $n=6$ independent experiments. Results were analysed using a 2-way ANOVA with the Holm-Sidak post hoc test. (B) To confirm that Piezo1 activation with Yoda1 had negligible effects on cellular stress, the JC-1 assay was performed to measure levels of mitochondrial stress in control and LPS-stimulated astrocytes. Neither Yoda1 nor GsMTx4 caused significant mitochondrial stress in LPS-treated astrocyte cultures. Data are presented as the mean absorbance levels (at $570 \mathrm{~nm}) \pm$ S.E.M normalised to control values $(\mathrm{n}=6$ independent experiments). Results were analysed using a 2-way ANOVA with the Holm-Sidak post hoc test. (C) Next, a 'wound healing' type cell migration assay was performed to assess changes in the speed of astrocyte migration in response to Piezo1 activation and inhibition. Activation of Piezo1 with Yoda1 did not significantly affect astrocyte migratory velocity in control (2.0 \pm 0.4 vs $3.3 \pm 1.0 \mu \mathrm{m} / \mathrm{h})$ or LPS-stimulated cultures $(1.4 \pm 0.5$ vs $2.3 \pm 0.3 \mu \mathrm{m} / \mathrm{h})$. Similarly, inhibition of Piezo1 with GsMTx4 did not alter astrocyte migration velocity in control (3.8 \pm 0.6 vs $3.3 \pm 1.0 \mu \mathrm{m} / \mathrm{h})$ or LPS-stimulated cultures $(5.0 \pm 0.9 \mathrm{vs} 2.3 \pm 0.3 \mu \mathrm{m} / \mathrm{h})$. However, there 
were significant differences in the velocities of LPS-stimulated astrocytes exposed to Yoda1 versus GsMTx4 $(1.4 \pm 0.5$ vs $5.0 \pm 0.9 \mu \mathrm{m} / \mathrm{h})$, suggesting that Piezo1 channel activation in reactive astrocytes inhibits the speed of collective cell migration. Data are presented as the mean distance travelled $(\mu \mathrm{m})$ in $48 \mathrm{~h}$ (black horizontal line), interquartile range (shaded rectangle) and the absolute distance values of $\mathrm{n}=6$ independent experiments. Differences were analysed using a 2-way ANOVA with the Holm-Sidak post hoc test and $* \mathrm{p}<0.05$. (D) and (E) are representative images of the migration assay showing (D) control and (E) LPS-stimulated astrocytes after $48 \mathrm{~h}$ treatment with GsMTx 4 . Scale bar $=100 \mu \mathrm{m}$. 

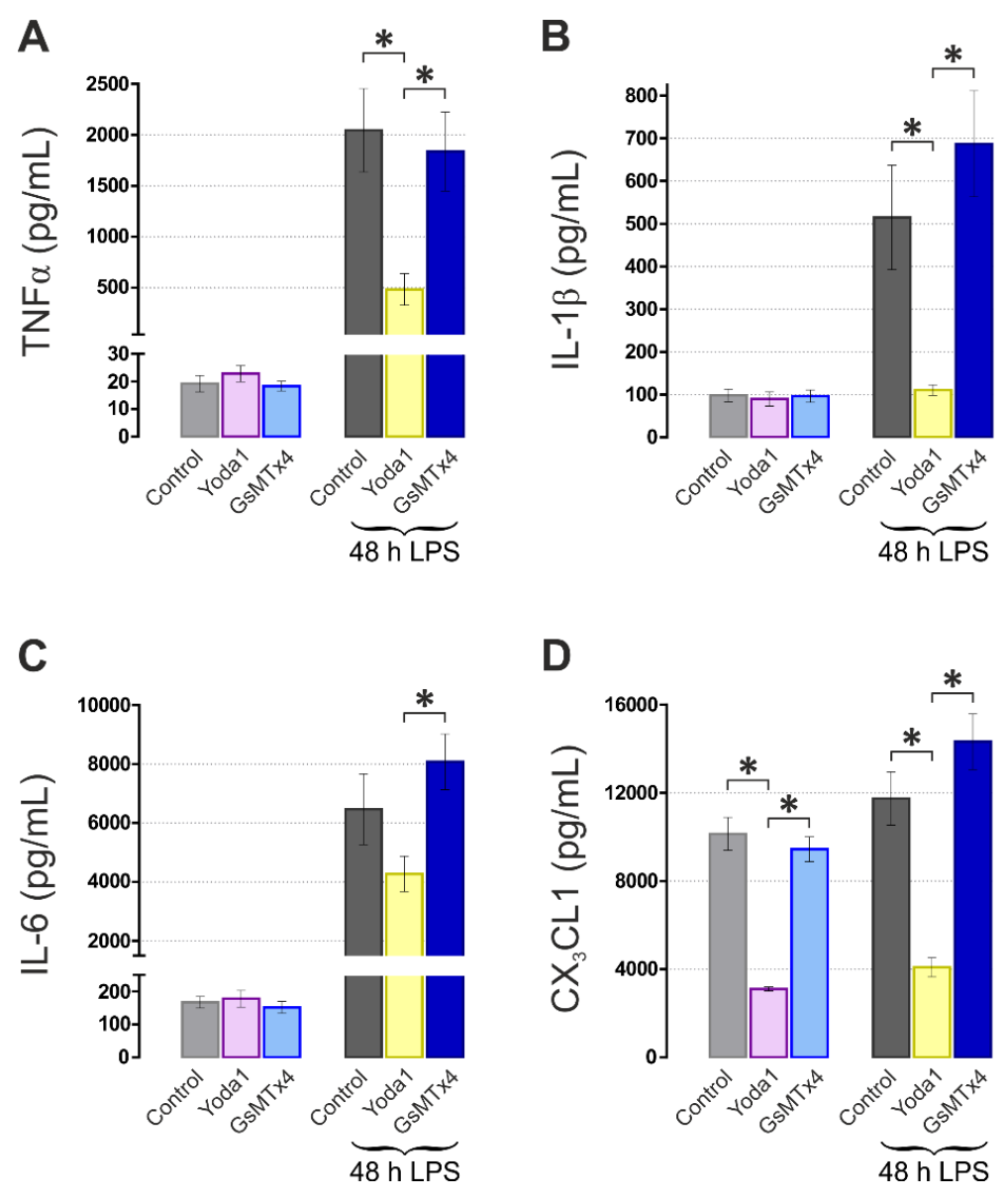

Figure 7: Piezo1 channels modulate cytokine and chemokine release from astrocytes. (A) Cytokine and chemokine release from control and LPS-stimulated astrocytes were measured after $48 \mathrm{~h}$ of either Yoda1 or GsMTx4 exposure. GsMTx4 peptide (500 nM) had little or no effect on cytokine release from reactive astrocytes. However, LPS-stimulated astrocytes exposed to $10 \mu \mathrm{M}$ Yoda1 for $48 \mathrm{~h}$ showed a significant decrease in (A) TNF $\alpha$ and (B) IL-1 $\beta$ release into the cell culture medium, as measured by ELISA. (C) Yoda1 did not cause a significant reduction in IL-6 release from astrocytes when compared to the LPS only group $(4,274 \pm 601$ vs $6,468 \pm 1,202 \mathrm{pg} / \mathrm{mL})$, but levels of IL-6 release in the LPS/Yoda1 group were significantly less than the LPS/GsMTx4-treated astrocytes $(8,076 \pm 947 \mathrm{pg} / \mathrm{mL})$. (D) Yoda1 also caused a significant decrease in $\mathrm{CX}_{3} \mathrm{CL} 1$ release from both control (3,105 \pm 94 vs 10,140 $\pm 741 \mathrm{pg} / \mathrm{mL})$ and LPS-stimulated astrocytes $(4,089 \pm 430$ vs $11,745 \pm 1,210 \mathrm{pg} / \mathrm{mL})$. Data from the ELISA assays are presented as mean \pm S.E.M. TNF $\alpha, I L-1 \beta$ and IL-6 ELISA's were repeated $n=5$ and $C_{3}$ CL1 ELISA was repeated $n=3$. Differences were analysed using a 2way ANOVA with the Holm-Sidak post hoc test and $* \mathrm{p}<0.05$. 


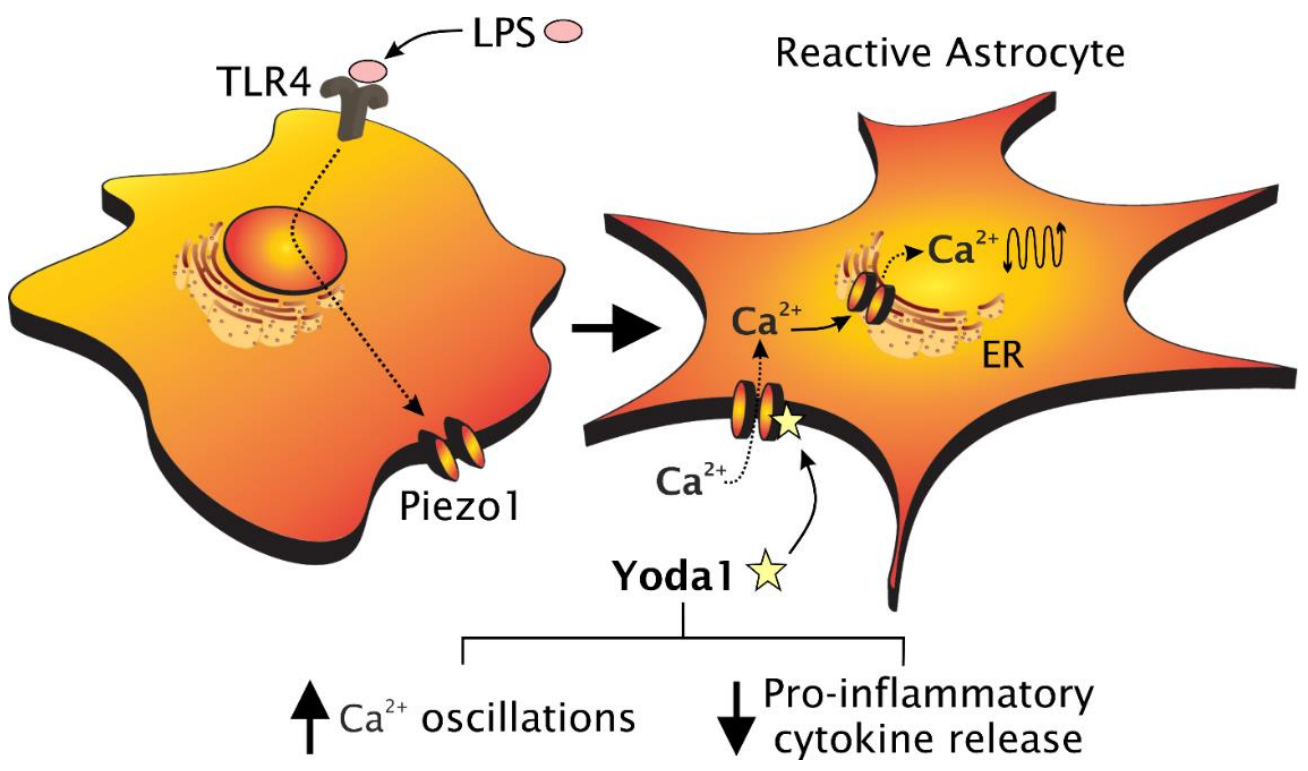

Figure 8: Proposed mechanism to explain Piezo1-mediated anti-inflammatory effects in LPS-stimulated astrocytes. Primary cortical astrocytes treated for $24 \mathrm{~h}$ with LPS upregulate Piezo1 expression. Activating Piezo1 channels with Yoda1 causes calcium $\left(\mathrm{Ca}^{2+}\right)$ influx and increases intracellular store-driven $\mathrm{Ca}^{2+}$ oscillations. In addition to its expression at the outer cell membrane, Piezo1 may localise to the endoplasmic reticulum (ER) of reactive astrocytes, thus enhancing intracellular $\mathrm{Ca}^{2+}$ release following Yoda1 exposure. Indeed, Syeda et al. (2015) have also reported that chelation of extracellular $\mathrm{Ca}^{2+}$ with EGTA did not completely abolish the calcium responses observed in HEK293T cells following Yoda1 stimulation. Alternatively, Yoda1 may also induce non-specific effects independent of Piezo1. In the absence of extracellular $\mathrm{Ca}^{2+}$, Yoda1 triggered release of $\mathrm{Ca}^{2+}$ from intracellular stores in reactive astrocytes (Fig. 5A) and increased $\mathrm{Ca}^{2+}$ oscillations compared to Yoda1-stimulated control astrocytes (Fig. 5B). Yoda1 also decreased IL-1 $\beta$, TNF $\alpha$ and $\mathrm{CX}_{3} \mathrm{CL} 1$ release from LPSstimulated astrocytes, suggesting that Piezo1 may play a role in dampening neuroinflammation in the brain. More work is needed to investigate if there is a link between Piezo1-mediated $\mathrm{Ca}^{2+}$ oscillations, reduced ATP-responsiveness and inhibition of pro-inflammatory cytokine release from reactive astrocytes. 


\section{References:}

Abbott, N. J., Ronnback, L., \& Hansson, E. (2006). Astrocyte-endothelial interactions at the blood-brain barrier. Nat Rev Neurosci, 7(1), 41-53. doi:10.1038/nrn1824

Adzic, M., Stevanovic, I., Josipovic, N., Laketa, D., Lavrnja, I., Bjelobaba, I. M., . . . Nedeljkovic, N. (2017). Extracellular ATP induces graded reactive response of astrocytes and strengthens their antioxidative defense in vitro. J Neurosci Res, 95(4), 1053-1066. doi:10.1002/jnr.23950

Bal-Price, A., Moneer, Z., \& Brown, G. C. (2002). Nitric oxide induces rapid, calciumdependent release of vesicular glutamate and ATP from cultured rat astrocytes. Glia, 40(3), 312-323. doi:10.1002/glia.10124

Blumenthal, N. R., Hermanson, O., Heimrich, B., \& Shastri, V. P. (2014). Stochastic nanoroughness modulates neuron-astrocyte interactions and function via mechanosensing cation channels. Proc Natl Acad Sci U S A, 111(45), 1612416129. doi:10.1073/pnas.1412740111

Bonkowski, D., Katyshev, V., Balabanov, R. D., Borisov, A., \& Dore-Duffy, P. (2011). The CNS microvascular pericyte: pericyte-astrocyte crosstalk in the regulation of tissue survival. Fluids Barriers CNS, 8(1), 8. doi:10.1186/2045-8118-8-8

Boue-Grabot, E., \& Pankratov, Y. (2017). Modulation of Central Synapses by AstrocyteReleased ATP and Postsynaptic P2X Receptors. Neural Plast, 2017, 9454275. doi:10.1155/2017/9454275

Bowers, H. C., Fiori, M. L., Khadela, J. B., Janmey, P. A., \& Galie, P. A. (2019). Cellmatrix tension contributes to hypoxia in astrocyte-seeded viscoelastic hydrogels composed of collagen and hyaluronan. Exp Cell Res, 376(1), 49-57. doi:10.1016/j.yexcr.2019.01.012

Buffo, A., Rolando, C., \& Ceruti, S. (2010). Astrocytes in the damaged brain: molecular and cellular insights into their reactive response and healing potential. Biochem Pharmacol, 79(2), 77-89. doi:10.1016/j.bcp.2009.09.014

Cahalan, S. M., Lukacs, V., Ranade, S. S., Chien, S., Bandell, M., Patapoutian, A. (2015). Piezo1 links mechanical forces to red blood cell volume. Elife, 4, e07370. doi: 10.7554/eLife.07370.

Cardenas, A., Kong, M., Alvarez, A., Maldonado, H., \& Leyton, L. (2014). Signaling pathways involved in neuron-astrocyte adhesion and migration. Curr Mol Med, 14(2), 275-290.

Chesnokova, V., Pechnick, R. N., \& Wawrowsky, K. (2016). Chronic peripheral inflammation, hippocampal neurogenesis, and behavior. Brain Behav Immun, 58, 1-8. doi:10.1016/j.bbi.2016.01.017

Choi, H. J., Sun, D., \& Jakobs, T. C. (2015). Astrocytes in the optic nerve head express putative mechanosensitive channels. Mol Vis, 21, 749-766.

Chubinskiy-Nadezhdin, V. I., Vasileva, V. Y., Vassilieva, I. O., Sudarikova, A. V., Morachevskaya, E. A., Negulyaev, Y. A. (2019) Agonist-induced Piezo1 activation suppresses migration of transformed fibroblasts. Biochem Biophys Res Commun. 514(1), 173-179. doi: 10.1016/j.bbrc.2019.04.139.

Copeland, C. S., Wall, T. M., Sims, R. E., Neale, S. A., Nisenbaum, E., Parri, H. R., \& Salt, T. E. (2017). Astrocytes modulate thalamic sensory processing via mGlu2 receptor activation. Neuropharmacology, 121, 100-110. doi:10.1016/j.neuropharm.2017.04.019

Dela Paz, N. G., \& Frangos J. A. (2018). Yoda1-induced phosphorylation of Akt and ERK1/2 does not require Piezo1 activation. Biochem Biophys Res Commun, 497(1) 220-225. doi:10.1016/j.bbrc.2018.02.058.

Dong, X. P., Wang, X., \& Xu, H. (2010) TRP channels of intracellular membranes. $J$ Neurochem, 113, 313-328. doi: 10.1111/j.1471-4159.2010.06626.x. 
Eisenhoffer, G. T., Loftus, P. D., Yoshigi, M., Otsuna, H., Chien, C. B., Morcos, P. A., \& Rosenblatt, J. (2012). Crowding induces live cell extrusion to maintain homeostatic cell numbers in epithelia. Nature, 484(7395), 546-549. doi:10.1038/nature10999

El-Hage, N., Podhaizer, E. M., Sturgill, J., \& Hauser, K. F. (2011) Toll-like receptor expression and activation in astroglia: differential regulation by HIV-1 Tat, gp120, and morphine. Immunol Invest, 40(5), 498-522. doi: 10.3109/08820139.2011.561904.

ElSheikh, M., Arani, A., Perry, A., Boeve, B. F., Meyer, F. B., Savica, R., Ehman, R. L., \& Huston, J. 3rd. (2017) MR Elastography Demonstrates Unique Regional Brain Stiffness Patterns in Dementias. AJR Am J Roentgenol, 209(2), 403-408. doi: 10.2214/AJR.16.17455.

Fellin, T., Pascual, O., Gobbo, S., Pozzan, T., Haydon, P. G., \& Carmignoto, G. (2004). Neuronal synchrony mediated by astrocytic glutamate through activation of extrasynaptic NMDA receptors. Neuron, 43(5), 729-743. doi:10.1016/j.neuron.2004.08.011

Filosa, J. A., \& Iddings, J. A. (2013). Astrocyte regulation of cerebral vascular tone. Am J Physiol Heart Circ Physiol, 305(5), H609-619. doi:10.1152/ajpheart.00359.2013

Friedl, P., \& Brocker, E. B. (2000). The biology of cell locomotion within threedimensional extracellular matrix. Cell Mol Life Sci, 57(1), 41-64. doi: $10.1007 / \mathrm{s} 000180050498$

Fujii, Y., Maekawa, S., \& Morita, M. (2017). Astrocyte calcium waves propagate proximally by gap junction and distally by extracellular diffusion of ATP released from volume-regulated anion channels. Sci Rep, 7(1), 13115. doi:10.1038/s41598017-13243-0

Fumagalli, M., Brambilla, R., D'Ambrosi, N., Volonte, C., Matteoli, M., Verderio, C., \& Abbracchio, M. P. (2003). Nucleotide-mediated calcium signaling in rat cortical astrocytes: Role of P2X and P2Y receptors. Glia, 43(3), 218-203. doi:10.1002/glia.10248

Gordon, G. R., Baimoukhametova, D. V., Hewitt, S. A., Rajapaksha, W. R., Fisher, T. E., \& Bains, J. S. (2005). Norepinephrine triggers release of glial ATP to increase postsynaptic efficacy. Nat Neurosci, 8(8), 1078-1086. doi:10.1038/nn1498

Gottlieb, P. A., \& Sachs, F. (2012). Piezo1: properties of a cation selective mechanical channel. Channels (Austin), 6(4), 214-219. doi:10.4161/chan.21050

Healy, L. M., Sheridan, G. K., Pritchard, A. J., Rutkowska, A., Mullershausen, F., \& Dev, K. K. (2013). Pathway specific modulation of S1P1 receptor signalling in rat and human astrocytes. Br J Pharmacol, 169(5), 1114-1129. doi:10.1111/bph.12207

Huang, Z., Wang, Y., Hu, G., Zhou, J., Mei, L., \& Xiong, W. C. (2016). YAP Is a Critical Inducer of SOCS3, Preventing Reactive Astrogliosis. Cereb Cortex, 26(5), 22992310. doi:10.1093/cercor/bhv292

Hung, W. C., Yang, J. R., Yankaskas, C. L., Wong, B. S., Wu, P. H., Pardo-Pastor, C., . . Konstantopoulos, K. (2016). Confinement Sensing and Signal Optimization via Piezo1/PKA and Myosin II Pathways. Cell Rep, 15(7), 1430-1441. doi:10.1016/j.celrep.2016.04.035

Jacob, F., Perez Novo, C., Bachert, C., \& Van Crombruggen, K. (2013). Purinergic signaling in inflammatory cells: P2 receptor expression, functional effects, and modulation of inflammatory responses. Purinergic Signal, 9(3), 285-306. doi:10.1007/s11302-013-9357-4

Jacques-Fricke, B. T., Seow, Y., Gottlieb, P. A., Sachs, F., \& Gomez, T. M. (2006). Ca2+ influx through mechanosensitive channels inhibits neurite outgrowth in opposition to other influx pathways and release from intracellular stores. $J$ Neurosci, 26(21), 5656-5664. doi:10.1523/jneurosci.0675-06.2006 
Kastritsis, C. H., Salm, A. K., \& McCarthy, K. (1992). Stimulation of the P2Y purinergic receptor on type 1 astroglia results in inositol phosphate formation and calcium mobilization. J Neurochem, 58(4), 1277-1284.

Koser, D. E., Thompson, A. J., Foster, S. K., Dwivedy, A., Pillai, E. K., Sheridan, G. K., . .. Franze, K. (2016). Mechanosensing is critical for axon growth in the developing brain. Nat Neurosci, 19(12), 1592-1598. doi:10.1038/nn.4394.

Lacroix, J. J., Botello-Smith, W. M., Luo Y. (2018) Probing the gating mechanism of the mechanosensitive channel Piezo1 with the small molecule Yoda1. Nat Commun, 9(1), 2029. doi: 10.1038/s41467-018-04405-3.

Leybaert, L., \& Sanderson, M. J. (2012). Intercellular Ca(2+) waves: mechanisms and function. Physiol Rev, 92(3), 1359-1392. doi:10.1152/physrev.00029.2011

Li, C., Rezania, S., Kammerer, S., Sokolowski, A., Devaney, T., Gorischek, A., . . . Schreibmayer, W. (2015). Piezo1 forms mechanosensitive ion channels in the human MCF-7 breast cancer cell line. Sci Rep, 5, 8364. doi:10.1038/srep08364

Li, J., Hou, B., Tumova, S., Muraki, K., Bruns, A., Ludlow, M. J., . . Beech, D. J. (2014). Piezo1 integration of vascular architecture with physiological force. Nature, 515(7526), 279-282. doi:10.1038/nature13701

Liddelow, S. A., Guttenplan, K. A., Clarke, L. E., Bennett, F. C., Bohlen, C. J., Schirmer, L., . . . Barres, B. A. (2017). Neurotoxic reactive astrocytes are induced by activated microglia. Nature, 541(7638), 481-487. doi:10.1038/nature21029

Lieberman, A. P., Pitha, P. M., Shin, H. S., \& Shin, M. L. (1989). Production of tumor necrosis factor and other cytokines by astrocytes stimulated with lipopolysaccharide or a neurotropic virus. Proc Natl Acad Sci U S A, 86(16), 63486352.

Liu, C. S. C., Raychaudhuri, D., Paul, B., Chakrabarty, Y., Ghosh, A. R., Rahaman, O., . . . Ganguly, D. (2018). Cutting Edge: Piezo1 Mechanosensors Optimize Human T Cell Activation. J Immunol, 200(4), 1255-1260. doi:10.4049/jimmunol.1701118

Maneshi, M. M., Sachs, F., \& Hua, S. Z. (2015). A Threshold Shear Force for Calcium Influx in an Astrocyte Model of Traumatic Brain Injury. J Neurotrauma, 32(13), 1020-1029. doi:10.1089/neu.2014.3677

Maroto, R., Kurosky, A., \& Hamill, O. P. (2012). Mechanosensitive $\mathrm{Ca}(2+)$ permeant cation channels in human prostate tumor cells. Channels (Austin), 6(4), 290-307. doi: $10.4161 /$ chan. 21063

Matias, I., Morgado, J., \& Gomes, F. C. A. (2019). Astrocyte Heterogeneity: Impact to Brain Aging and Disease. Front Aging Neurosci, 11, 59. doi:10.3389/fnagi.2019.00059

McHugh, B. J., Buttery, R., Lad, Y., Banks, S., Haslett, C., \& Sethi, T. (2010). Integrin activation by Fam38A uses a novel mechanism of R-Ras targeting to the endoplasmic reticulum. J Cell Sci, 123(Pt 1), 51-61. doi:10.1242/jcs.056424

McHugh, B. J., Murdoch, A., Haslett, C., \& Sethi, T. (2012). Loss of the integrin-activating transmembrane protein Fam38A (Piezo1) promotes a switch to a reduced integrindependent mode of cell migration. PLoS One, 7(7), e40346. doi:10.1371/journal.pone.0040346

Metea, M. R., \& Newman, E. A. (2006). Glial cells dilate and constrict blood vessels: a mechanism of neurovascular coupling. $J$ Neurosci, 26(11), 2862-2870. doi:10.1523/jneurosci.4048-05.2006

Moroni, M., Servin-Vences, M. R., Fleischer, R., Sanchez-Carranza, O., \& Lewin, G. R. (2018). Voltage gating of mechanosensitive PIEZO channels. Nat Commun, 9(1), 1096. doi:10.1038/s41467-018-03502-7

Moshayedi, P., Costa Lda, F., Christ, A., Lacour, S. P., Fawcett, J., Guck, J., \& Franze, K. (2010). Mechanosensitivity of astrocytes on optimized polyacrylamide gels analyzed by quantitative morphometry. J Phys Condens Matter, 22(19), 194114. doi:10.1088/0953-8984/22/19/194114 
Moshayedi, P., Ng, G., Kwok, J. C., Yeo, G. S., Bryant, C. E., Fawcett, J. W., . . Guck, J. (2014). The relationship between glial cell mechanosensitivity and foreign body reactions in the central nervous system. Biomaterials, 35(13), 3919-3925. doi:10.1016/j.biomaterials.2014.01.038

Nakano, Y., Furube, E., Morita, S., Wanaka, A., Nakashima, T., \& Miyata, S. (2015). Astrocytic TLR4 expression and LPS-induced nuclear translocation of STAT3 in the sensory circumventricular organs of adult mouse brain. J Neuroimmunol, 278, 144-158. doi:10.1016/j.jneuroim.2014.12.013

Nishizaki, T. (2004). ATP- and adenosine-mediated signaling in the central nervous system: adenosine stimulates glutamate release from astrocytes via A2a adenosine receptors. J Pharmacol Sci, 94(2), 100-102.

Nourse, J. L., \& Pathak, M. M. (2017). How cells channel their stress: Interplay between Piezo1 and the cytoskeleton. Semin Cell Dev Biol, 71, 3-12. doi:10.1016/j.semcdb.2017.06.018

O'Sullivan, S. A., Velasco-Estevez, M., \& Dev, K. K. (2017). Demyelination induced by oxidative stress is regulated by sphingosine 1-phosphate receptors. Glia, 65(7), 1119-1136. doi:10.1002/glia.23148

Pathak, M. M., Nourse, J. L., Tran, T., Hwe, J., Arulmoli, J., Le, D. T., . . Tombola, F. (2014). Stretch-activated ion channel Piezo1 directs lineage choice in human neural stem cells. Proc Natl Acad Sci U S A, 111(45), 16148-16153. doi:10.1073/pnas.1409802111

Pau, G., Fuchs, F., Sklyar, O., Boutros, M., \& Huber, W. (2010). EBImage--an R package for image processing with applications to cellular phenotypes. Bioinformatics, 26(7), 979-981. doi:10.1093/bioinformatics/btq046

Pavel, M., Renna, M., Park, S. J., Menzies, F. M., Ricketts, T., Fullgrabe, J., . . . Rubinsztein, D. C. (2018). Contact inhibition controls cell survival and proliferation via YAP/TAZ-autophagy axis. Nat Commun, 9(1), 2961. doi:10.1038/s41467-018-05388-x

Prakriya, M. (2009) The molecular physiology of CRAC channels. Immunol Rev. 231, 8898. doi: 10.1111/j.1600-065X.2009.00820.x.

Pougnet, J. T., Toulme, E., Martinez, A., Choquet, D., Hosy, E., \& Boue-Grabot, E. (2014). ATP P2X receptors downregulate AMPA receptor trafficking and postsynaptic efficacy in hippocampal neurons. Neuron, 83(2), 417-430. doi:10.1016/j.neuron.2014.06.005

Rocha, D. N., Ferraz-Nogueira, J. P., Barrias, C. C., Relvas, J. B., \& Pego, A. P. (2015). Extracellular environment contribution to astrogliosis-lessons learned from a tissue engineered 3D model of the glial scar. Front Cell Neurosci, 9, 377. doi:10.3389/fncel.2015.00377

Rorth, P. (2009). Collective cell migration. Annu Rev Cell Dev Biol, 25, 407-429. doi:10.1146/annurev.cellbio.042308.113231

Rothhammer, V., \& Quintana, F. J. (2015). Control of autoimmune CNS inflammation by astrocytes. Semin Immunopathol, 37(6), 625-638. doi:10.1007/s00281-015-05153

Scemes, E., \& Giaume, C. (2006). Astrocyte calcium waves: what they are and what they do. Glia, 54(7), 716-725. doi:10.1002/glia.20374

Serrano, A., Haddjeri, N., Lacaille, J. C., \& Robitaille, R. (2006). GABAergic network activation of glial cells underlies hippocampal heterosynaptic depression. $J$ Neurosci, 26(20), 5370-5382. doi:10.1523/jneurosci.5255-05.2006.

Sethi, M. K., \& Zaia, J. (2017) Extracellular matrix proteomics in schizophrenia and Alzheimer's disease. Anal Bioanal Chem, 409(2), 379-394. doi: 10.1007/s00216016-9900-6. 
Simen, A. A., Bordner, K. A., Martin, M. P., Moy, L. A., \& Barry, L. C. (2011). Cognitive dysfunction with aging and the role of inflammation. Ther Adv Chronic Dis, 2(3), 175-195. doi:10.1177/2040622311399145

Sofroniew, M. V., \& Vinters, H. V. (2010). Astrocytes: biology and pathology. Acta Neuropathol, 119(1), 7-35. doi:10.1007/s00401-009-0619-8

Song, Y., Li, D., Farrelly, O., Miles, L., Li, F., Kim, S. E., . . Jan, Y. N. (2019). The Mechanosensitive Ion Channel Piezo Inhibits Axon Regeneration. Neuron, 102(2), 373-389.e376. doi:10.1016/j.neuron.2019.01.050

Syeda, R., Xu, J., Dubin, A. E., Coste, B., Mathur, J., Huynh, T., Matzen, J., Lao, J., Tully, D. C., Engels, I. H., Petrassi, H. M., Schumacher, A. M., Montal, M., Bandell, M., Patapoutian, A. (2015) Chemical activation of the mechanotransduction channel Piezo1. Elife, 4, e07369. doi: 10.7554/eLife.07369.

Tyler, W. J. (2012) The mechanobiology of brain function. Nat. Rev. Neurosci. 13(12), 867-878. doi: 10.1038/nrn3383.

Velasco-Estevez, M., Mampay, M., Boutin, H., Chaney, A., Warn, P., Burgess, E., . . . Sheridan, G. K. (2018). Infection augments expression of mechanosensing Piezo1 channels in amyloid plaque-reactive astrocytes. Front. Aging Neurosci, 10, 332 doi:10.3389/fnagi.2018.00332

Verkhratsky, A., \& Parpura, V. (2014). Store-operated calcium entry in neuroglia. Neurosci Bull, 30, 125-133. doi: 10.1007/s12264-013-1343-x.

Wang, S., Chennupati, R., Kaur, H., Iring, A., Wettschureck, N., \& Offermanns, S. (2016). Endothelial cation channel PIEZO1 controls blood pressure by mediating flowinduced ATP release. J Clin Invest, 126(12), 4527-4536. doi:10.1172/jci87343

Yang, X. N., Lu, Y. P., Liu, J. J., Huang, J. K., Liu, Y. P., Xiao, C. X., . . Guleng, B. (2014). Piezo1 is as a novel trefoil factor family 1 binding protein that promotes gastric cancer cell mobility in vitro. Dig Dis Sci, 59(7), 1428-1435. doi:10.1007/s10620-014-3044-3

Zhan, J. S., Gao, K., Chai, R. C., Jia, X. H., Luo, D. P., Ge, G., . . Yu, A. C. (2017). Astrocytes in Migration. Neurochem Res, 42(1), 272-282. doi:10.1007/s11064016-2089-4 